A Organisation des Nations Unies pour I'alimentation et l'agriculture

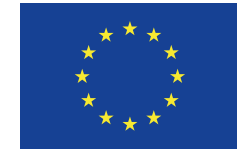

Union Européenne cirad

LARECHERCHE AGRONOMIQUE POURLE DÉVELOPPEMENT

\title{
PROFIL DES SYSTÈMES ALIMENTAIRES - MADAGASCAR
}

Activer la transformation durable et inclusive de nos systèmes alimentaires

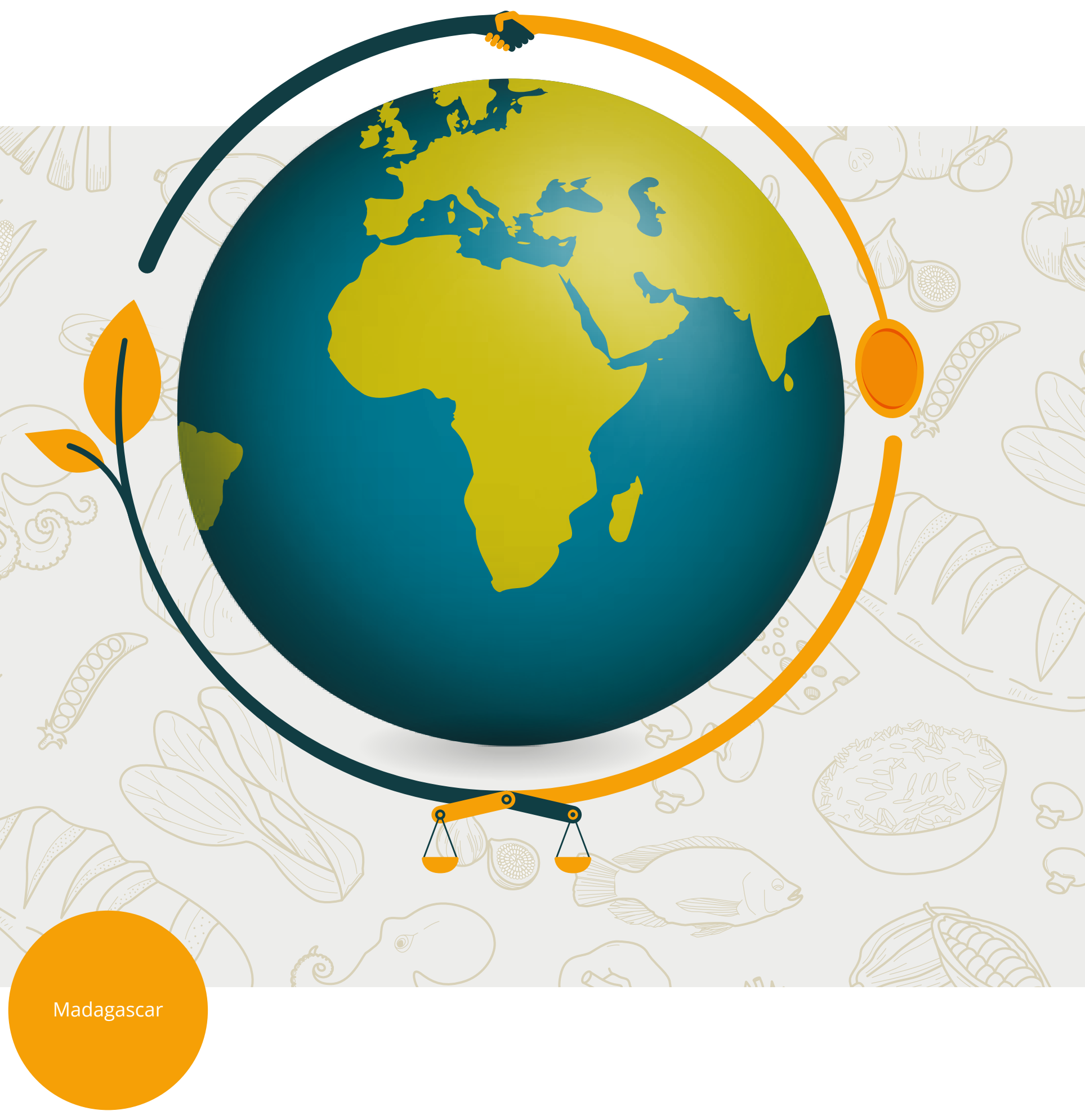




\section{PROFIL DES SYSTÈMES ALIMENTAIRES - MADAGASCAR}

Activer la transformation durable et inclusive de nos systèmes alimentaires

Publié par

I'Organisation des Nations Unies pour l'alimentation et l'agriculture,

le Centre de coopération international en recherche agronomique pour le développement,

et l'Union européenne.

Rome, Montpellier, Bruxelles, 2021. 
Citer comme suit:

FAO, Union européenne et Cirad. 2021. Profil des systèmes alimentaires - Madagascar. Activer la transformation durable et inclusive de nos systèmes alimentaires. Rome, Bruxelles et Montpellier, France.

https://doi.org/10.4060/cb6861fr

Les appellations employées dans ce produit d'information et la présentation des données qui y figurent n'impliquent de la part de l'Organisation des Nations Unies pour l'alimentation et l'agriculture (FAO), du Centre de coopération international en recherche agronomique pour le développement (Cirad) de l'Union européenne (UE) aucune prise de position quant au statut juridique ou au stade de développement des pays, territoires, villes ou zones ou de leurs autorités, ni quant au tracé de leurs frontières ou limites. Les lignes pointillées sur les cartes représentent des frontières approximatives dont le tracé peut ne pas avoir fait l'objet d'un accord définitif. Le fait qu'une société ou qu'un produit manufacturé, breveté ou non, soit mentionné ne signifie pas que la FAO, le Cirad ou l'UE approuvent ou recommandent ladite société ou ledit produit de préférence à d'autres sociétés ou produits analogues qui ne sont pas cités.

ISBN [FAO] 978-92-5-134979-3

(C) FAO, 2021

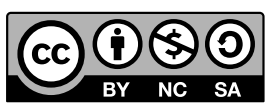

Certains droits réservés. Cette œuvre est mise à la disposition du public selon les termes de la Licence Creative Commons - Attribution - Pas d'Utilisation Commerciale - Partage dans les Mêmes Conditions 3.0 Organisations Intergouvernementales (CC BY-NC-SA 3.0 IGO; https://creativecommons.org/licenses/by-nc-sa/3.0/igo/legalcode.fr).

Selon les termes de cette licence, cette œuvre peut être copiée, diffusée et adaptée à des fins non commerciales, sous réserve que la source soit mentionnée. Lorsque l'œuvre est utilisée, rien ne doit laisser entendre que la FAO cautionne tels ou tels organisation, produit ou service. L'utilisation du logo de la FAO n'est pas autorisée. Si l'œuvre est adaptée, le produit de cette adaptation doit être diffusé sous la même licence Creative Commons ou sous une licence équivalente. Si l'œuvre est traduite, la traduction doit obligatoirement être accompagnée de la mention de la source ainsi que de la clause de non-responsabilité suivante: «La traduction n'a pas été réalisée par l'Organisation des Nations Unies pour l'alimentation et l'agriculture (FAO). La FAO n'est pas responsable du contenu ni de l'exactitude de la traduction. L'édition originale [langue] est celle qui fait foi.»

Tout litige relatif à la présente licence ne pouvant être résolu à l'amiable sera réglé par voie de médiation et d'arbitrage tel que décrit à l'Article 8 de la licence, sauf indication contraire contenue dans le présent document. Les règles de médiation applicables seront celles de l'Organisation mondiale de la propriété intellectuelle (http://www.wipo.int/amc/fr/mediation/ rules) et tout arbitrage sera mené conformément au Règlement d'arbitrage de la Commission des Nations Unies pour le droit commercial international (CNUDCI).

Matériel attribué à des tiers. Il incombe aux utilisateurs souhaitant réutiliser des informations ou autres éléments contenus dans cette oeuvre qui y sont attribués à un tiers, tels que des tableaux, des figures ou des images, de déterminer si une autorisation est requise pour leur réutilisation et d'obtenir le cas échéant la permission de l'ayant-droit. Toute action qui serait engagée à la suite d'une utilisation non autorisée d'un élément de l'œuvre sur lequel une tierce partie détient des droits ne pourrait l'être qu'à l'encontre de l'utilisateur.

Ventes, droits et licences. Les produits d'information de la FAO sont disponibles sur le site web de la FAO (www.fao.org/ publications) et peuvent être obtenus sur demande adressée par courriel à: publications-sales@fao.org. Les demandes visant un usage commercial doivent être soumises à: www.fao.org/contact-us/licence-request. Les questions relatives aux droits et aux licences doivent être adressées à: copyright@fao.org. 


\section{Chers acteurs investis,}

La non durabilité des systèmes alimentaires reste manifeste à Madagascar. Nous observons encore des situations répétées d'insécurité alimentaire dans de nombreuses parties du pays.

Les systèmes alimentaires englobent une palette très large d'actions humaines impactant d'une façon ou d'une autre l'alimentation, générant d'importants enjeux culturels ainsi que des défis économiques, sociaux et environnementaux.

Aujourd'hui, Madagascar cherche à infléchir sa trajectoire pour garantir à sa population, la disponibilité et l'accès à une alimentation diversifiée, sûre, nutritive et abordable. Il est également primordial de créer des emplois et des moyens de subsistance décents, de protéger les ressources naturelles et la biodiversité, et de s'assurer l'équilibre et l'équité entre les différents territoires et régions.

Un chantier énorme, ambitieux mais réalisable nous attend. Aussi, il est fondamental de s'enquérir de la multi - dimensionnalité des enjeux entourant les systèmes alimentaires nationaux, leur interdépendance et leur hétérogénéité spatiale.

À cet effet, je tiens à saluer l'initiative conjointe de l'Organisation des Nations Unies pour l'alimentation et l'agriculture - FAO et du Centre de coopération internationale en recherche agronomique pour le développement - Cirad, avec l'appui de l'Union européenne, pour ce formidable travail accompli sur le diagnostic rapide des systèmes alimentaires à Madagascar. Un diagnostic qui rentre dans le cadre de la mise en œuvre de la stratégie « de la ferme à la table » dont Madagascar est un des premiers pays bénéficiaires.

Ce premier travail constitue une bonne base de réflexion à nos dialogues nationaux que nous allons mener et qui vont nous permettre d'annoncer des solutions concrètes et d'en partager au prochain Sommet des Nations Unies sur les systèmes alimentaires.

À noter qu'impulser un nouveau contour aux systèmes alimentaires va demander bien plus que des initiatives agronomiques. Les efforts entrepris en faveur de la production ont certes contribué à accroître la disponibilité alimentaire, mais trouvent rapidement leur limite dans la paupérisation des ménages ruraux, les carences des régimes alimentaires, l'enclavement de nombreuses zones productrices, l'insécurité rurale et la dégradation des ressources naturelles.

Le défi de vouloir améliorer les systèmes alimentaires nationaux dépend à la fois des efforts du secteur agricole pour la disponibilité en quantité et qualité d'aliments diversifiés, des travaux publics pour le désenclavement des zones de production, de l'éducation pour que les consommateurs d'aujourd'hui aient les comportements nutritionnels adaptés, et des forces de sécurité. Mais il dépend aussi du secteur du commerce pour l'approvisionnement et la distribution ainsi que du secteur de l'environnement pour la préservation et la durabilité des ressources naturelles.

Je fais alors appel au renforcement des initiatives communes multisectorielles et à une pensée systémique basée sur la transversalité et la multisectorialité de toutes les parties prenantes ayant des responsabilités et engagements vis-à-vis des systèmes alimentaires à Madagascar: le secteur public, le secteur privé, l'organisation des sociétés civiles, les chercheurs, les producteurs, et les consommateurs.

Ensemble, investissons pour la durabilité, la résilience et la performance de nos systèmes alimentaires.

RANARIVELO Lucien Fanomezantsoa, Ministre de l'Agriculture, de l'Élevage et de la Pêche - MADAGASCAR 


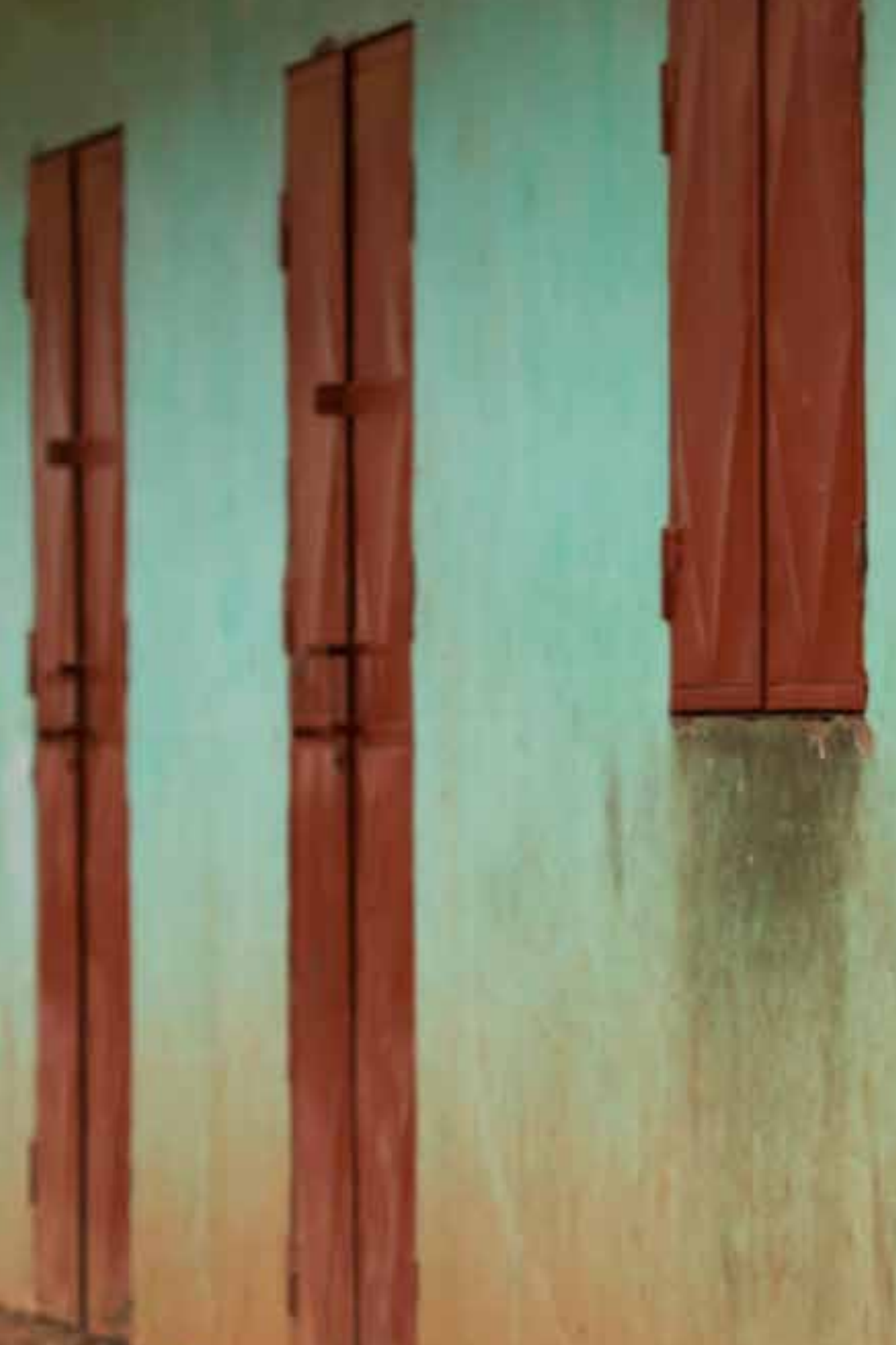




\section{PROFIL DES SYSTÈMES ALIMENTAIRES MADAGASCAR}

\section{Messages clés}

La non durabilité du système alimentaire malagasy est perçue à travers l'insécurité alimentaire qui prévaut dans la quasi-totalité de lîle, la dégradation des ressources naturelles tant au niveau des terres qu'au niveau de la biodiversité et de l'eau, les inégalités territoriales et sociales engendrées non seulement par une répartition naturelle inégale des ressources mais aussi par une répartition non appropriée des interventions de l'État et des organismes non-gouvernementaux dans tout le territoire, ainsi que la pauvreté généralisée dans toute l'île. La durabilité du système alimentaire dépendrait alors de la capacité à relever les huit défis suivants:

Figure 1. Les messages clés pour un système alimentaire durable à Madagascar

Les habitudes alimentaires et

l'éducation nutritionelle

contribuent à une alimentation très peu diversifiée et à une faible attention à la qualité des aliments

La capacité d'investissement dans la production est limitée par les très fortes contraintes financières des ménages agricoles

La quantité et la qualité consommées sont limitées par un pouvoir d'achat très faible

Les ressources humaines et financières trop limitées allouées au secteur, généralement gérées au niveau central, limitent les initiatives locales

Les modèles économiques de chaînes de valeur incitent les producteurs à vendre leurs produits, au détriment de leur propre consommation alimentaire

Un accès limité et inégal au foncier, aux services, aux ressources, à l'éducation contraint fortement les exploitations.
La croissance démographique accentue la pression foncière dans les bas-fonds (déjà intense sur les Hautes Terres) menaçant la viabilité de l'agriculture familiale

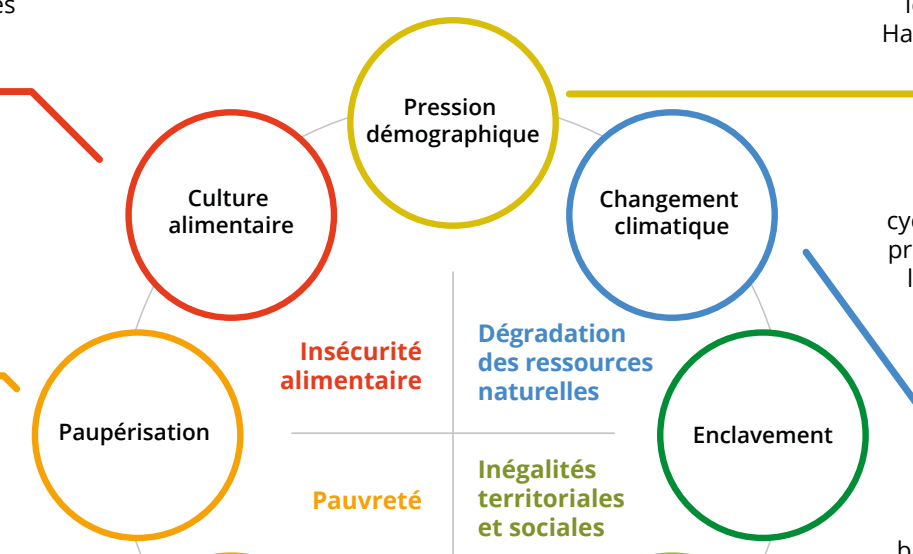

L'instabilité croissante du climat (retard des pluies, sécheresse, cyclones) accentue l'instabilité de la production et contribue à dégrader les infrastructures. Elle induit une pression croissante sur les ressources forestières, pour compenser les baisses de rendement.

L'enclavement pénalise les producteurs, y compris dans des bassins de production majeurs, en les privant d'accès aux services, en limitant leur accès aux marchés et en favorisant l'insécurité. II limite la valorisation de nouveaux espaces.

L'insécurité contraint la production à rester au niveau «subsistance», en induisant des stratégies anti-risques. Elle freine la valorisation de vastes zones faiblement peuplées. 
L'aspect systémique du problème alimentaire est important, de plus la coordination des actions en réponse aux problèmes de différents niveaux (national, régional et local) constitue une condition de réussite pour une amélioration des conditions nutritionnelles et alimentaires à Madagascar.

\section{Chiffres clés}

\begin{tabular}{|c|c|}
\hline Population totale (2019) $1: 25,7$ millions & Accès à l'électricité (2017)2: 24,1\% \\
\hline Croissance démographie (2019)1:2,66\% & Abonnements téléphone mobile (2017): 91\% \\
\hline Croissance de la population urbaine (2019)': 4,44\% & Indice d'écart global entre les sexes $(\mathbf{2 0 2 0})^{4}: 0,725$ \\
\hline $\begin{array}{l}\text { Indice de Gini (2014)1: 42,6 } \\
\text { PIB/hab. (2018)1: } 1678 \text { USD PPP } 2010\end{array}$ & $\begin{array}{l}\text { Pop. en âge de travailler ayant au moins une } \\
\text { éducation primaire (2017)5: } 74 \%\end{array}$ \\
\hline $\begin{array}{l}\text { \% de l'agriculture, la forêt et la pêche dans le PIB } \\
\text { (2018)2 } 2 \text { : } 24 \%\end{array}$ & Superficie forestière $(\mathbf{2 0 1 5})^{6}: 21,4 \%$ de la sup. totale \\
\hline $\begin{array}{l}\text { Indice d'efficacité du gouvernement, entre }-2,5 \text { et + } \\
2,5(2019)^{3}:-1,1\end{array}$ & \\
\hline
\end{tabular}

Sources: (1) INSTAT/RGPH; (2) Indicateurs de la Banque mondiale; (3) Banque mondiale, Indicateurs de gouvernance mondiaux; (4) Forum économique mondial; (5) ILOSTAT; (6) UNSTAT (Indicateurs des ODD). 


\section{Secteur agricole et alimentaire: chiffres clés et tendances}

\section{La production agricole augmente mais sa progression peine à suivre la croissance démographique soutenue. Cette situation entraîne une dépendance croissante aux importations, notamment pour les céréales, le sucre et les corps gras. En termes de valeur, la balance commerciale alimentaire est néanmoins rééquilibrée par les exportations centrées sur des produits de très forte valeur comme les épices (vanille, clou de girofle) et le cacao.}

La production agricole malgache est très diversifiée (produits amylacés, viande et produits animaux, fruits et légumes...) et elle est orientée à plus de 90 pour cent vers le marché domestique. Le riz occupe la première place en volume, mais en valeur, l'élevage tient une place encore plus importante (lait, viande bovine, porcine et volaille).

Malgré une augmentation de la production agricole, sa progression n'arrive pas à suivre la croissance démographique soutenue de 2 à 3 pour cent par an. Pour les productions végétales, une embellie passagère est observée pour le riz, le maïs, le manioc au début des années 2000, mais la production est en chute depuis 2013.

Pour les produits de la pêche et de l'aquaculture, après une stagnation de la production entre 2008 et 2013, la production augmente progressivement mais un besoin d'augmentation de la production est estimé nécessaire (environ 10000 tonnes entre 2016 et 2020) dans une hypothèse de maintien du niveau de

Figure 2. Structure de la production (\% de la valeur)

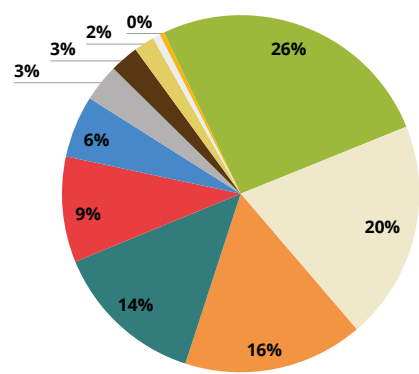

\footnotetext{
Viande Riz Autres produits amylacés Lait \& œufs

Fruits et légumes Sucres Epices —Café et cacao Graines export Non alimentaire Miel
}

consommation par habitant et de la croissance démographique (Banque mondiale 2016).

Quant à la production animale, seuls les œufs et la volaille ont suivi la croissance démographique, jusqu'au début des années 2000. La production de viande de bœuf comme de lait stagnent. Le porc a été durement affecté par la peste porcine à la fin des années 90 et la production chute à nouveau depuis 2019 .

L'insuffisance de la production résulte de divers facteurs: (1) faible productivité à l'hectare corrélée avec un faible accès des producteurs aux services et une faible fertilité des sols, (2) faible superficie cultivée en raison de la fragmentation des exploitations (63 pour cent des agriculteurs exploitent moins de 1,5 ha) et/ou par manque d'accessibilité des superficies cultivables, tout cela dans un contexte d'insécurité foncière et publique quasigénéralisée. Des chocs récurrents (cyclones, invasions acridiennes, sécheresses, inondations...) aggravent ces difficultés structurelles.

Figure 3. Évolution des principales productions végétales (en indice)

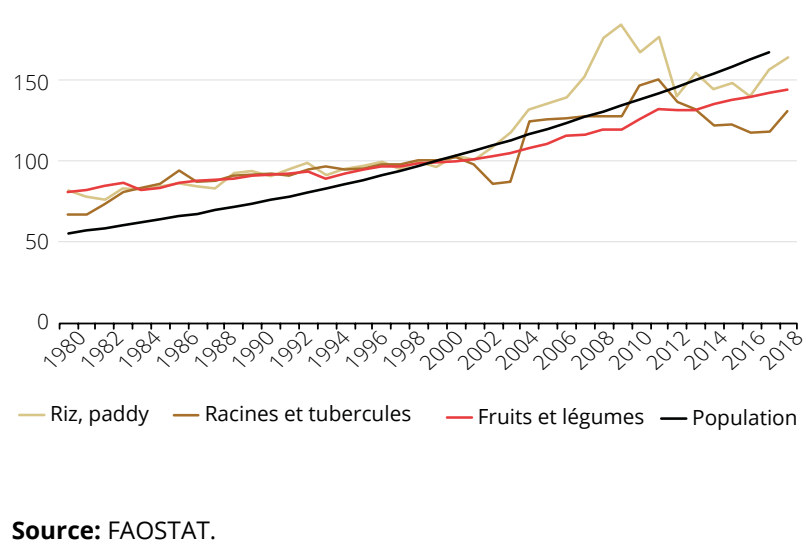


En conséquence, la dépendance aux importations s'accroit; elle est particulièrement marquée pour les céréales (passant de 10 pour cent sur la période 2010-2013 à 23 pour cent sur la période 20162018), le sucre et les corps gras. À titre d'illustration, le pays a importé 532000 tonnes de riz et 312000

Figure 4. Évolution de la production animale, en indice

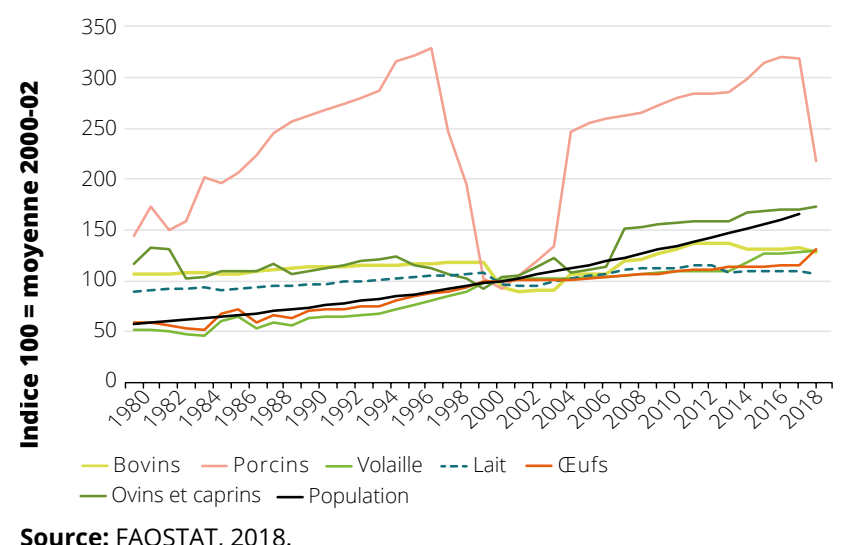

tonnes de blé en 2019. En termes de valeur, la balance commerciale alimentaire est néanmoins rééquilibrée par les exportations centrées sur des produits de très forte valeur comme les épices (vanille, clou de girofle) et le cacao. Ces exportations représentent 1,6 fois les importations alimentaires.
Figure 5. Évolution des importations (en valeur), principaux groupes de produits

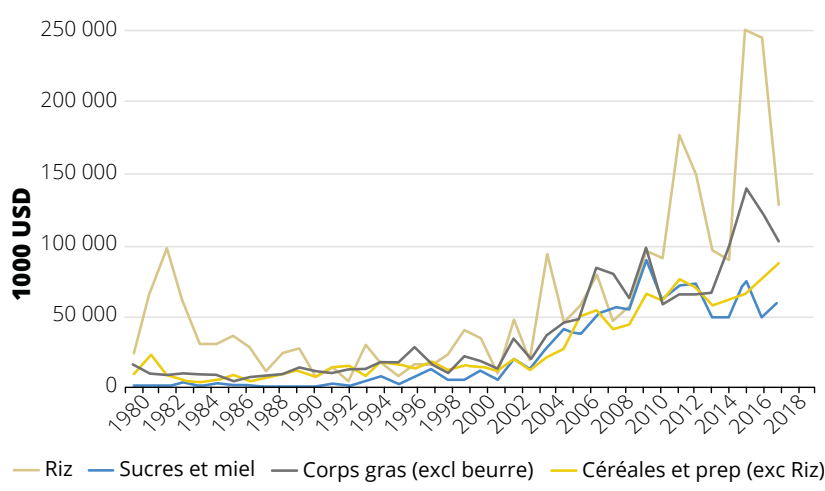

Source: FAOSTAT, 2018.

\section{Consommation alimentaire: chiffres clés et tendances Un régime alimentaire centré sur les céréales et les racines amylacées}

Le bilan alimentaire en 2018 (calculé sur la base de FAOSTAT) indique une disponibilité énergétique moyenne de $1476 \mathrm{kcal} / \mathrm{pers} . / \mathrm{j}$,

Figure 6. Disponibilité alimentaire par groupe de produits

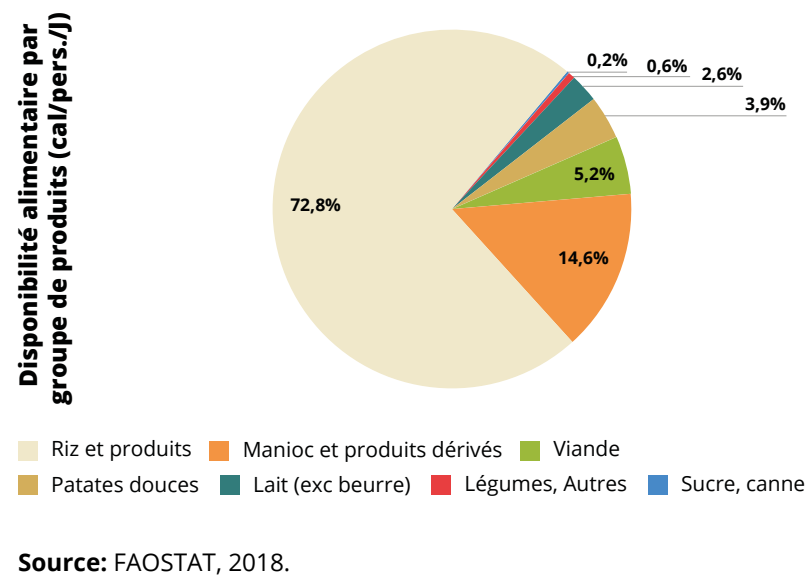

soit un bilan déficitaire par rapport au besoin minimal moyen estimé à $2133 \mathrm{kcal} / \mathrm{jour} /$ personne. Le régime alimentaire est très

Figure 7. Structure de la consommation selon les revenus

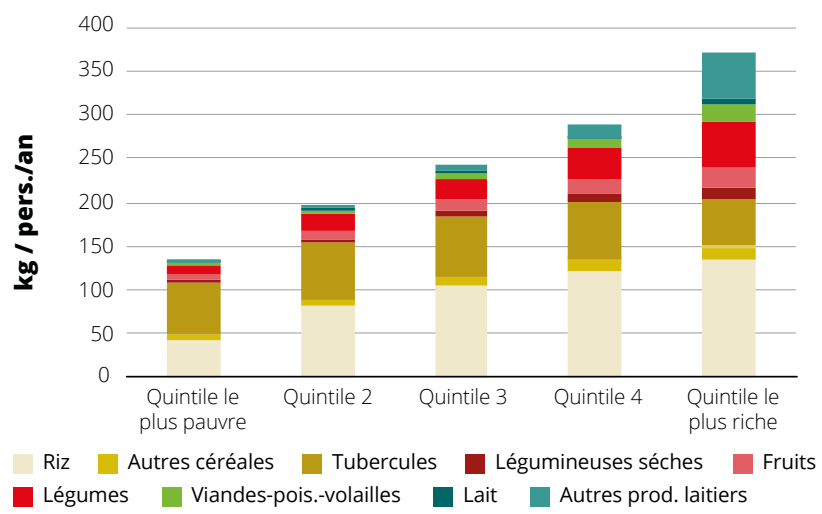

Source: INSTAT / EPM, 2010. 
fortement basé sur les céréales - principalement le riz- et les racines amylacées (manioc et patate douce) dont les disponibilités par personne par jour, en 2017, étaient respectivement de 462 et 365 g; et est pauvre en protéines animales (38 $\mathrm{g} /$ pers./j de viande, $14 \mathrm{~g}$ de poissons et fruits de mer et $58 \mathrm{~g}$ de lait et œufs) et en fruits et légumes (161 g/pers./j).

Une réelle diversité alimentaire n'est observée que chez 20 pour cent des ménages aux plus hauts revenus.

\section{Quelle performance du système alimentaire vis-à-vis des quatre objectifs fondamentaux d'un système alimentaire durable?}

\section{Sécurité alimentaire, nutrition, santé}

Le système alimentaire peine à répondre au premier objectif d'assurer la sécurité alimentaire et nutritionnelle contribuant à la santé de la population malagasy. En 2019, Madagascar figure au $114^{\mathrm{e}}$ rang sur 117 pays selon l'Indice de la faim dans le monde et au $108^{\mathrm{e}}$ rang sur 113 pays sur I'Indice global de sécurité alimentaire.

En 2016-2018, la sous-alimentation concernait 44 pour cent de la population et sévissait particulièrement dans le sud du pays. La situation s'est fortement dégradée depuis 2013. La malnutrition chez les enfants de moins de 5 ans, avec de fortes disparités régionales, est également préoccupante: en 2016, 42 pour cent des enfants de moins de 5 ans souffrent d'un retard de croissance, situation affectant notamment les Hautes Terres centrales et l'est.

Les causes de cette faible performance sont multidimensionnelles: une production agricole

Figure 8. Évolution de quelques indicateurs clés
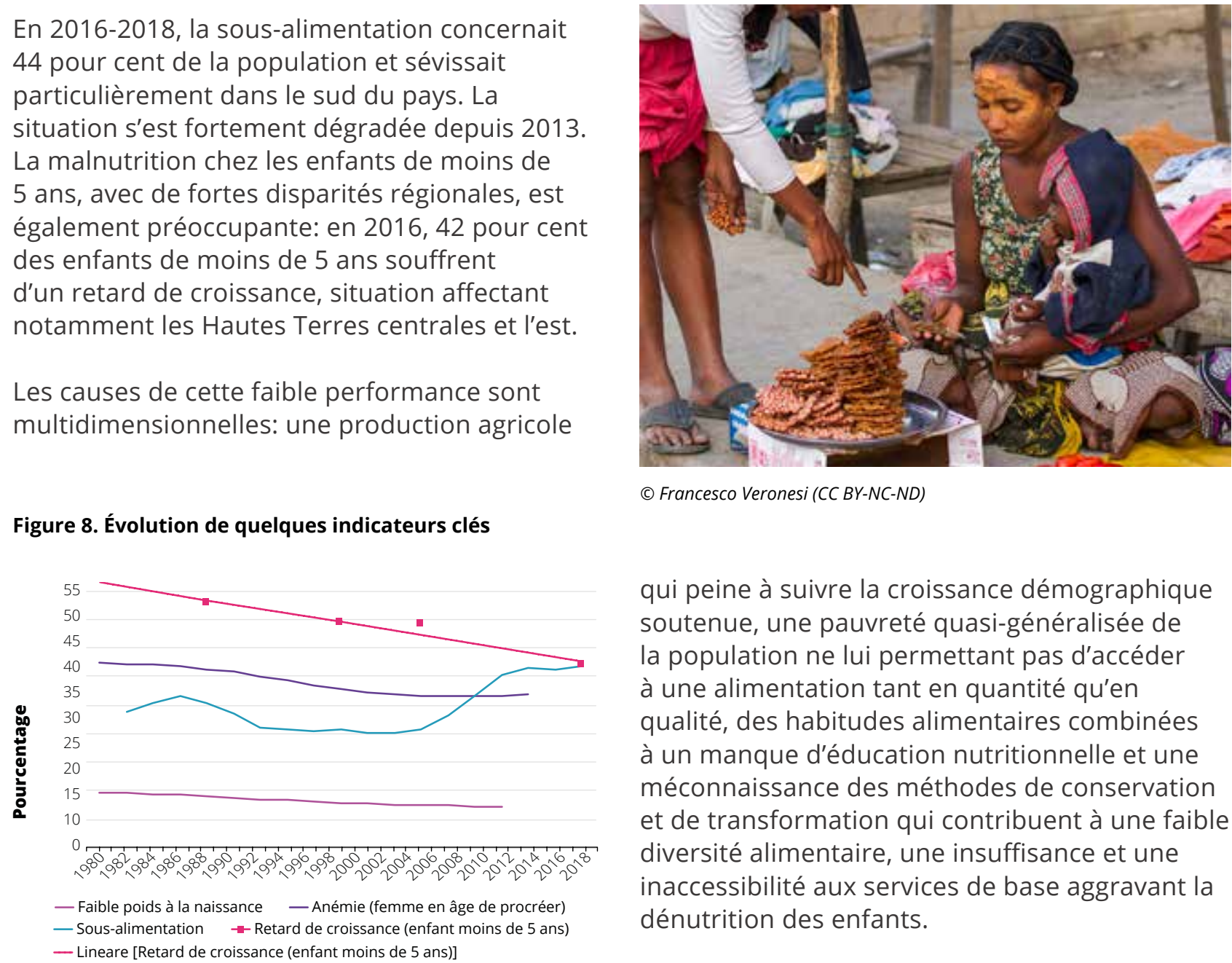

(c) Francesco Veronesi (CC BY-NC-ND)

qui peine à suivre la croissance démographique soutenue, une pauvreté quasi-généralisée de la population ne lui permettant pas d'accéder à une alimentation tant en quantité qu'en qualité, des habitudes alimentaires combinées à un manque d'éducation nutritionnelle et une méconnaissance des méthodes de conservation et de transformation qui contribuent à une faible diversité alimentaire, une insuffisance et une inaccessibilité aux services de base aggravant la dénutrition des enfants.

Source: FAOSTAT, 2018. 
Figure 9. Le système alimentaire par rapport à la dimension sécurité alimentaire, nutrition et santé

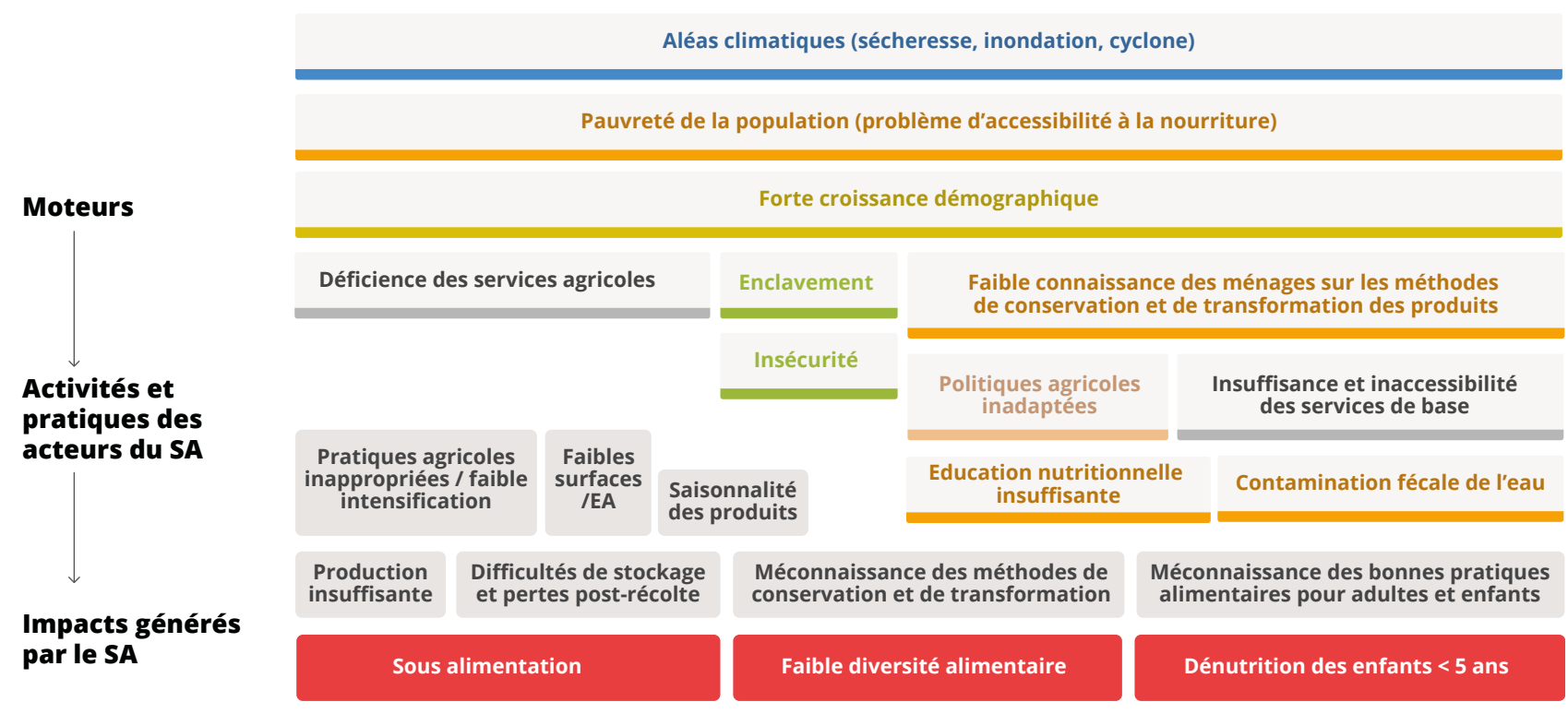

Source: les auteurs.

\section{Socioéconomie}

Le système alimentaire malagasy ne permet pas aux ménages agricoles, en particulier les petites exploitations agricoles familiales, très majoritaires, de sortir de la pauvreté.

Le secteur agricole, majoritairement alimentaire, constitue le principal pourvoyeur d'emplois (68 pour cent de la population active). Mais il ne génère que de faibles revenus pour les ménages agricoles, ne leur permettant pas d'investir dans leurs exploitations agricoles. De ce fait, le secteur de l'agriculture, la forêt et la pêche contribue relativement faiblement au PIB (24 pour cent). Outre les facteurs expliquant la faiblesse de la production agricole, la mauvaise répartition de la valeur ajoutée au détriment des producteurs due à une asymétrie d'information et à une faible organisation des chaînes de valeur contribuent à la faiblesse des revenus des ménages agricoles.

La volatilité des prix des produits agricoles constitue un facteur aggravant dans la mesure où les producteurs sont dans la plupart des cas obligés de vendre à bas prix à la récolte puis d'acheter à prix fort en période de soudure. Cette volatilité pénalise également les consommateurs. A titre d'illustration, le riz paddy enregistre une variation intra-annuelle jusqu'à 40 pour cent (FAO, MAEP, PAM, 2019), 333 pour cent pour le manioc séché et 300 pour cent pour le maïs (FEWSNET, 2020). Cette variation est plus importante en milieu rural qu'au niveau des centres urbains.

Figure 10. Contributions des différents secteurs à la croissance du PIB

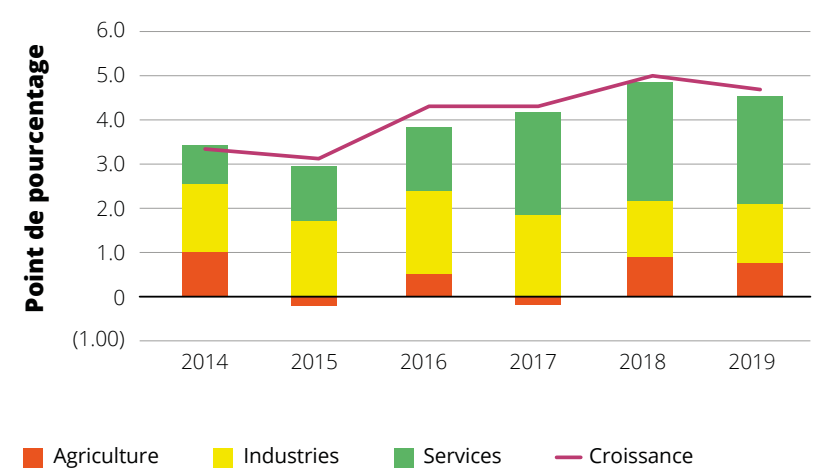

Source: Banque mondiale, 2019. 
Figure 11. Le système alimentaire par rapport à la dimension socioéconomique

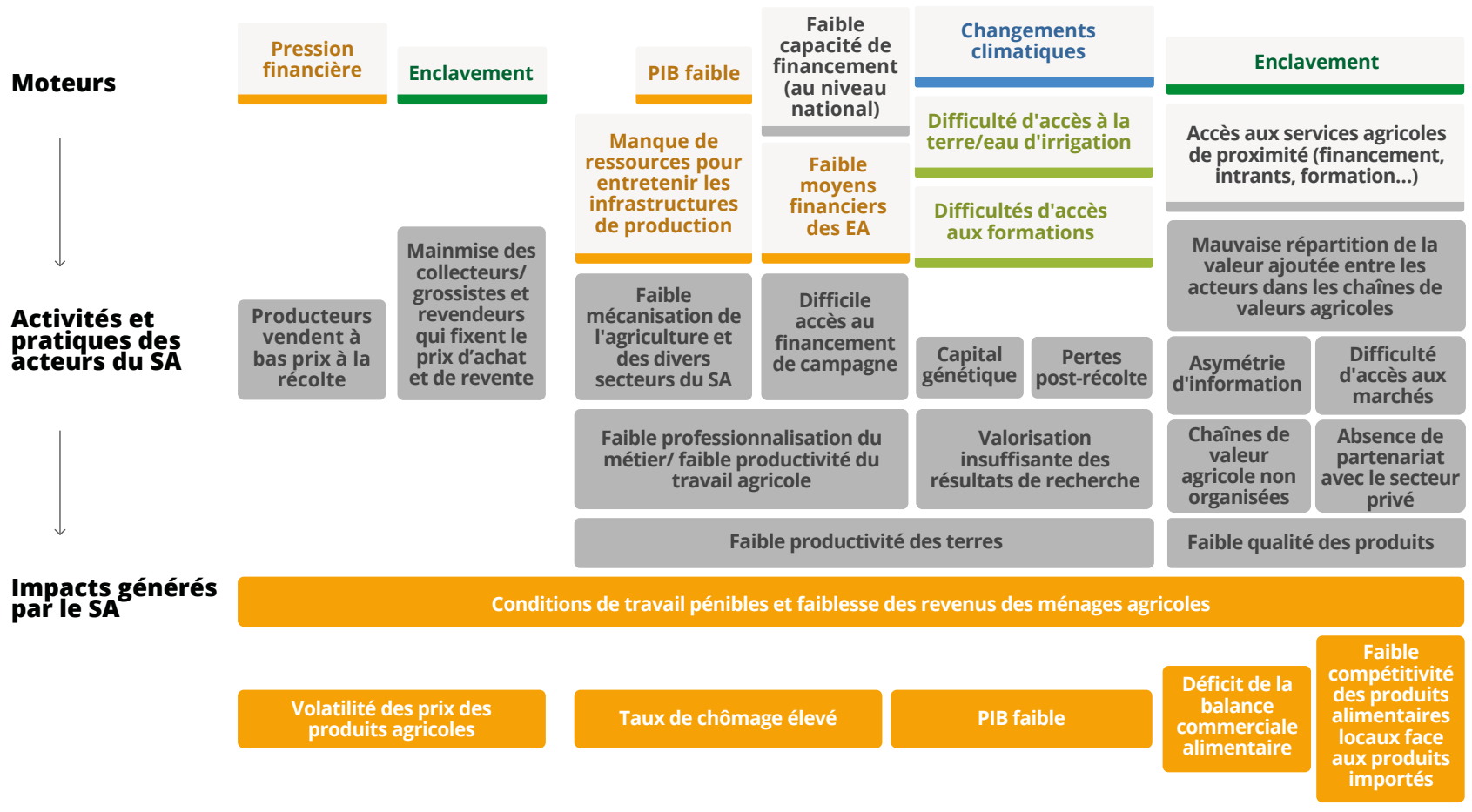

Source: les auteurs.

\section{Équilibre territorial et équité \\ Un mécanisme engagé de décentralisation qui peine à exploiter la diversité régionale au profit d'un système alimentaire durable}

Actuellement, les collectivités locales

décentralisées manquent de ressources et manquent aussi de pouvoir, car l'État est encore fortement centralisé. Les décisions importantes sont prises au niveau du gouvernement central et la synergie intersectorielle est presque inexistante au niveau local. Ceci pénalise l'adéquation des actions aux spécificités du territoire et réduit énormément leur efficacité.

Au niveau du système alimentaire, les déséquilibres entre territoires et entre acteurs se traduisent par (1) l'enclavement presque généralisé des zones rurales: les coûts d'entretien sont rarement supportés par les structures décentralisées, les coûts de transport deviennent plus importants tant pour l'écoulement des produits que pour l'approvisionnement en nourriture et en intrants nécessaires à la production; (2) les différents problèmes fonciers: l'insécurité foncière limite les investissements du producteur; (3) le déséquilibre entre les acteurs du système alimentaire: l'accès aux informations économiques et commerciales est limité à cause des limites communicationnelles au niveau rural et de l'inégalité éducationnelle, et la faible capacité de stockage limite le pouvoir de négociation des producteurs; (4) la distribution inégale des ressources publiques: le processus d'attribution des deniers publics est décidé au niveau des instances nationales, les actions vis-à-vis du système alimentaire considérées comme priorité locale font face à des difficultés de financement; (5) des échanges entre régions entravés par les aléas climatiques et l'état des infrastructures, qui isolent les territoires avec de fortes insécurités alimentaires. 
Figure 12. Le système alimentaire par rapport à la dimension équilibre territorial et équité

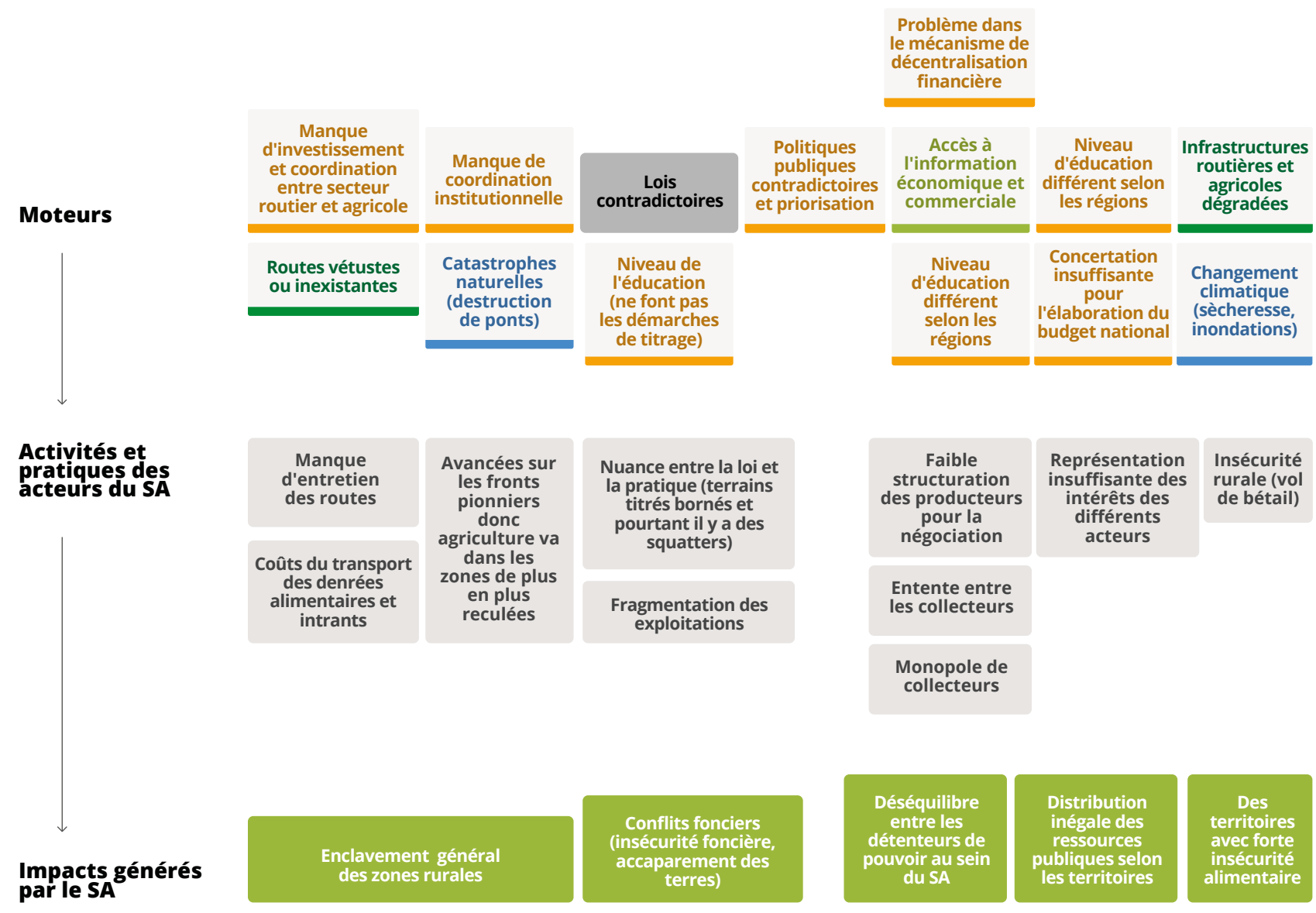

Source: les auteurs.

\section{Environnement et ressources naturelles \\ Le système alimentaire éprouve des difficultés à gérer, préserver et régénérer les écosystèmes et les ressources naturelles.}

La déforestation, accentuée ces dernières années, est directement impactée par le difficile accès aux terres fertiles du fait des contraintes foncières, de la faiblesse des rendements, la faible capacité d'investissement des ménages ruraux, la pression du marché du bois, la disponibilité en essences de qualité et la fluctuation des prix des produits agricoles sur le marché international (Rabefarihy A.T. , 2013). À cause de leur faible niveau technique et matériel, les producteurs malagasy sont dans une spirale de faible productivité, et la forêt offre (surtout hors des aires protégées) un accès aux terres fertiles pour l'extension de la superficie de production. Les pratiques agroécologiques offrent une alternative face au souci d'intensification (productivité par unité de surface), mais elles sont encore émergentes et sont surtout observées dans les zones où des projets de développement sur l'agroécologie ont été développés (centre, centre-ouest et sud-est).

Concernant la dégradation physique des sols, les pratiques agricoles non durables (culture sur pente très forte, culture sur brûlis) favorisent la perte de la partie de matière organique 
et d'éléments minéraux essentiels. En outre, le phénomène cyclique de feux de brousse fait que les matières organiques du sol ont presque disparu et qu'un climat érosif suffit pour décaper le sol et former les lavaka. Ces derniers donnent un sol impropre à l'agriculture en amont mais aussi un ensablement des aménagements en aval.

La demande croissante des villes en produits alimentaires, notamment maraîchers, nécessite une intensification de la production qui passe souvent par l'utilisation d'engrais chimiques pour compenser la faible qualité des sols latéritiques. Mais les doses prescrites sont rarement suivies par les producteurs, causant une dégradation de la qualité chimique des sols. D'autre part, la lutte contre les maladies des cultures devient de plus en plus importante face à l'avènement de nouveaux ravageurs et nouveaux insectes. La mauvaise utilisation des pesticides (dont certains, interdits depuis des années, sont toujours utilisés) accélère la pollution chimique du sol, et du fait de dosages et périodes d'application inadaptés, restent présents dans les aliments.

Le déclin des ressources halieutiques est le résultat de la dégradation des habitats marins, principalement due à la coupe illégale des mangroves où se régénèrent une partie des espèces marines, à la destruction des coraux, notamment causée par les violents cyclones et à la surpêche (pêche industrielle illicite et utilisation de maillages non réglementaires). Dans le nord-ouest, les forêts de mangrove sont illégalement coupées à cause du charbonnage.

Le déclin des stocks des ressources halieutiques fait suite à une forte croissance démographique, à la dégradation des habitats côtiers et marins, aux effets du changement climatique sur les activités de pêche et à la faible valorisation des produits. En conséquence, le dynamisme des milieux côtiers malgaches est dans un cercle vicieux de dégénérescence où le manque d'activités alternatives génératrices de revenus et la précarité se conjuguent.

Figure 13. Le système alimentaire par rapport à la dimension environnement

\section{Moteurs}

$$
\downarrow
$$

\section{Activités et} pratiques des
acteurs du SA

$$
\downarrow
$$

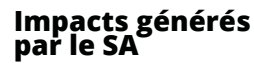

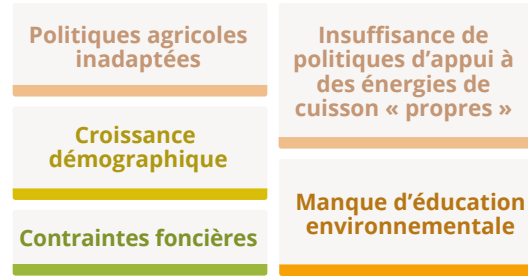

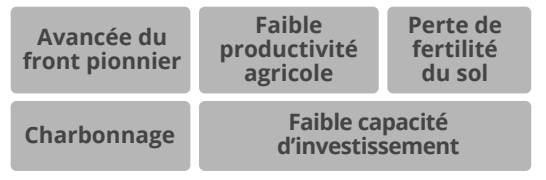

Déforestation
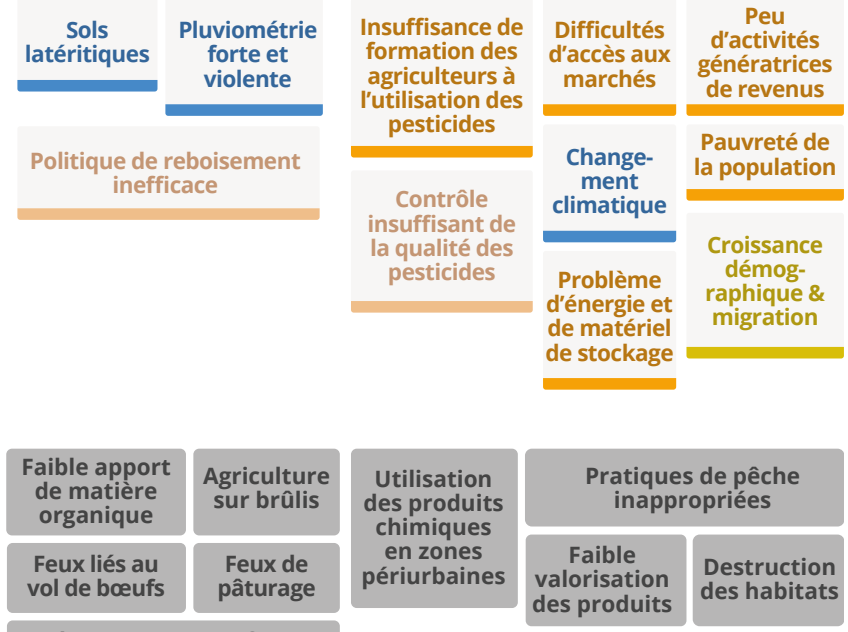

Cultures sur pentes fortes 


\section{Quels sont les principaux programmes et politiques publiques qui affectent la durabilité du système alimentaire?}

La Politique générale de l'État (PGE, 2020) définit les principales orientations pour les dimensions affectant la durabilité du système alimentaire et se traduit par le Plan Émergence Madagascar (PEM 2019-2023). Les finalités sont de permettre l'accélération et la stabilité de la croissance économique dans le pays, de stimuler l'épanouissement de sa potentialité économique, d'améliorer la protection du climat des affaires, d'assurer la bonne gouvernance, d'améliorer la redevabilité de l'administration, de moderniser Madagascar par de nouvelles technologies, d'assurer la couverture en énergie dans tout le pays, d'améliorer les conditions de vie de la population et d'assurer la protection de l'environnement. Ainsi, les actions qui vont être initiées au niveau des secteurs sont multiples. Dans le secteur agricole, les actions suivantes sont en cours actuellement: (1) le Programme sectoriel agriculture élevage pêche/Plan national d'investissement agricole (2016-2020), (2) la Stratégie nationale de développement de l'agriculture biologique, (3) la Stratégie nationale de développement de l'agribusiness. Dans le secteur santé et nutrition, il y a lieu de mentionner: (1) le Plan national d'action pour la nutrition III 2017-2021, (2) le Programme national d'alimentation, de nutrition et de santé scolaire (PNANSS II). Dans le secteur de la gouvernance et de l'équilibre territorial: (1) la Lettre de politique de décentralisation et de déconcentration (LP2D 2004), (2) le Programme national de décentralisation et de déconcentration (PN2D 2005), (3) la Lettre de politique de décentralisation emergente (adoptée en conseil des ministres en mai 2021). Dans le secteur de l'environnement: (1) le Plan d'action national de lutte contre le changement climatique (PANLCC, 2019), (2) le Programme environnemental pour le développement durable (2016), (3) la Politique forestière de Madagascar (PFM, 2017), (4) le Plan directeur forestier national et directives des actions de reboisement (2019-2029), (5) la Stratégie et les plans d'action nationaux pour la biodiversité (SPANB).

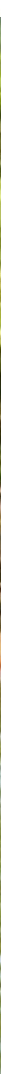




\section{Zonage des systèmes alimentaires}

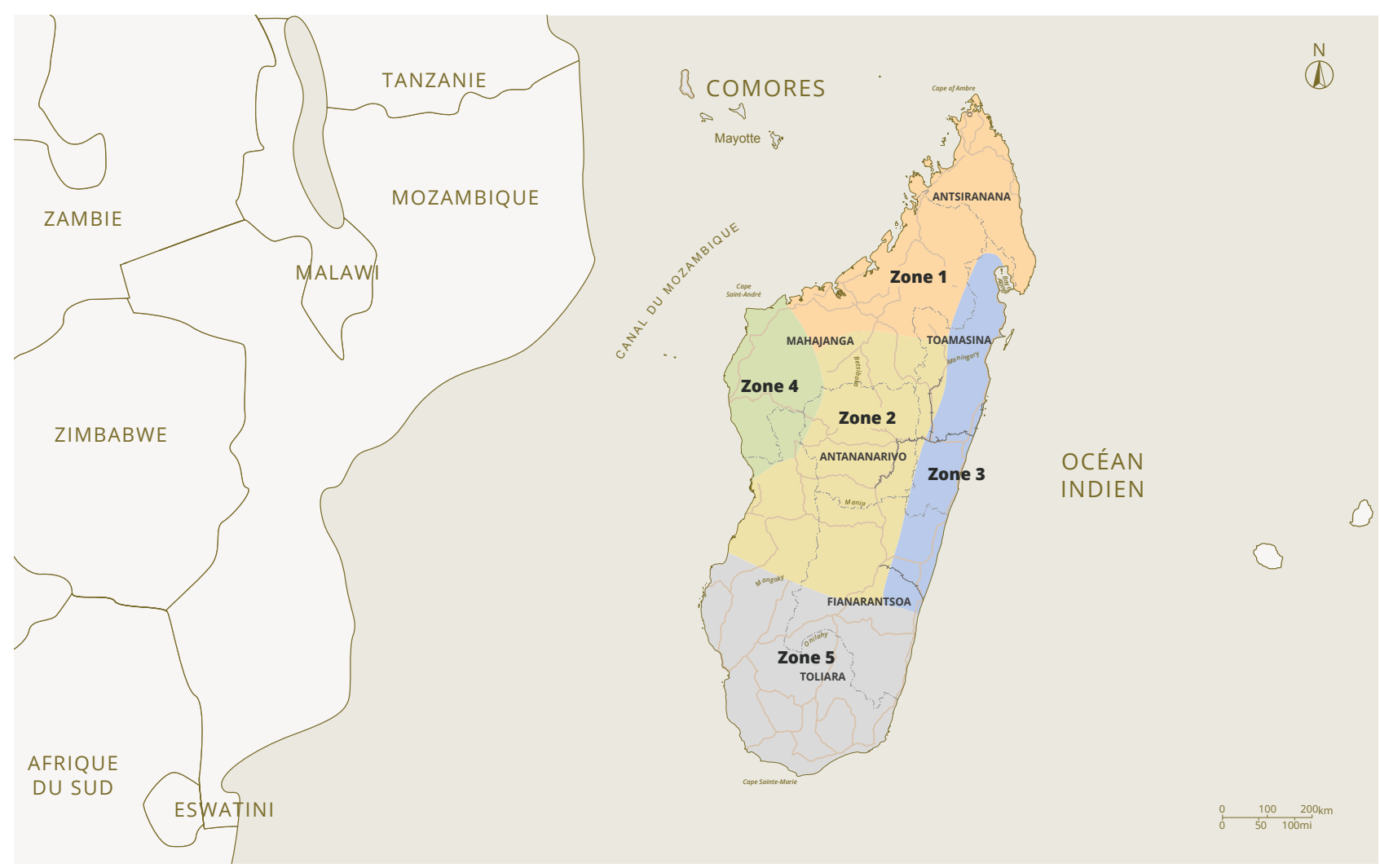

Source: section de l'information géospatiale de I'ONU.

\section{Zone 1: le Grand Nord}

Une zone favorisée: plus de diversité climatique, moins peuplée, un littoral et des parcs nationaux très attractifs pour le tourisme.

La zone 1 se situe dans le nord du pays: régions Diana, Sava, Sofia et la partie septentrionale de Boeny.

Cette zone est portée sur les cultures de rentes destinées au marché national ou international (vanille, cacao, ylang-ylang, canne à sucre, tabac, oignon et plus récemment black eyes - ou niébé), sur le tourisme (Nosy-be, Antsiranana, Mahajanga), sur la production rizicole (Bealanana et Befandriana, Marovoay et Andapa), sur l'élevage bovin ( 24,5 pour cent du cheptel national de zébus avec près de deux millions de têtes - MAEP/DGE,
2019), sur la pêche industrielle, l'aquaculture de crevettes et la pêche traditionnelle avec environ 19000 pêcheurs.

Les principaux défis sont: (1) l'enclavement des bassins de production (Bealanana, Sava); (2) la gestion durable des ressources naturelles et la perte de biodiversité des forêts qui subissent les activités de charbonnage au niveau des mangroves, de l'exploitation des bois précieux et de l'extension des terres agricoles (Sava); (3) l'insuffisance de la production vivrière à cause de l'attractivité d'activités plus rémunératrices (vanille, bois de rose, prostitution, etc.); (4) les apports financiers significatifs mais instables issus des cultures de rente (vanille, cacao), de la pêche et de la crevetticulture, contribuant à des taux de pauvreté relativement 
faibles même si la répartition de la valeur se fait au détriment des producteurs et en faveur des entreprises de transformation, des industries et des commerçants; (5) le vieillissement des plantations (vanilliers, cacaoyers, ylang-ylang, etc.) qui va à court et moyen terme diminuer la productivité et la compétitivité des produits et qui nécessite un accompagnement des petits producteurs pour garantir le maintien de la qualité; (6) l'ensablement et l'accentuation des phénomènes de lavakisation dans les zones rizicoles qui diminuent les surfaces utiles et font baisser la fertilité des sols; (7) la baisse des stocks de poissons; la malnutrition infantile, malgré l'autosuffisance en riz et la diversité des productions végétales, animales et halieutiques et la pauvreté relativement faible de la zone; (8) l'harmonisation des activités entre pêcheurs traditionnels et pêcheurs industriels.

Les leviers identifiés pour atteindre la durabilité du système alimentaire dans la zone 1 sont:

(1) la promotion de la transformation et l'amélioration des infrastructures de stockage; (2) la remise en état des routes; (3) le renforcement des services agricoles et le renforcement des capacités des producteurs; (4) la revalorisation du métier d'agriculteur (population jeune) et les formations ciblées (notamment cultures de rentes); (5) l'éducation nutritionnelle des familles; (6) les actions en faveur d'une répartition équitable de la valeur ajoutée tout au long de la chaîne (accent à mettre sur les producteurs); (7) la gestion de la fertilité des sols pour augmenter la production; et (8) la formalisation des secteurs informels et sécurité d'investissement tout en valorisant les investisseurs locaux.

\section{Zone 2: le Grand Centre}

Le poumon vivrier et le plus grand bassin de consommation du pays

Géographiquement, la zone 2 est située dans le centre du pays (régions Haute Matsiatra, Amoron'i Mania, Menabe, Vakinankaratra, Analamanga, Alaotra Mangoro, Betsiboka, Itasy, Bongolava).
Bien qu'abritant la capitale du pays, la population de la zone est à 65-95 pour cent rurale. Les exploitations agricoles sont majoritairement de type familial sur de petites parcelles. Des formes modernes de fermes mécanisées existent mais elles sont assez dispersées et se limitent souvent à de nouveaux projets d'investissement à capitaux étrangers.

Le riz occupe la première place des spéculations vivrières paysannes devant le manioc, le maïs et la patate douce. Le haricot tient le premier rang pour les légumineuses tandis que l'arachide constitue la principale source de matière grasse végétale de la zone. La gamme des cultures maraîchères est très large et la zone est le plus gros fournisseur de légumes frais pour les zones 3 et 1. Le climat permet une production étendue de fruits tempérés sur les hautes terres (pommes, prunes, oranges, etc.). La population tire aussi des revenus substantiels de l'élevage bovin et laitier, de la porciculture, de l'aviculture et enfin de la pêche et de la pisciculture grâce à la multitude de zones humides et de plans d'eau qui parsèment la zone. L'élevage d'embouche est extensif sur les vastes espaces de la partie ouest et devient semi-extensif vers le centre pour la production laitière et même en stabulation fixe autour des villes. La partie littorale (Menabe) de cette zone abrite les pêcheurs industriels d'export et les pêcheurs traditionnels approvisionnant la zone en poissons et produits de mer. La pisciculture est présente surtout dans I'Itasy et l'Amoron'i Mania pour les besoins du marché local.

En outre, cette zone est la plus industrialisée (industrie agro-alimentaire surtout) du pays avec des transformateurs dans la biscuiterie, les fruits et légumes, les boissons, la confiserie, les produits laitiers.

La zone du Grand Centre fait face aux défis suivants: (1) la mauvaise répartition de la valeur ajoutée au détriment des producteurs et en faveur des entreprises de transformation, des industriels et des commerçants; (2) la volatilité des prix au niveau des producteurs; une 
pauvreté importante en particulier dans le sud de la zone 2; (3) une malnutrition infantile persistante; (4) une faible qualité sanitaire et nutritionnelle des produits frais suite à un usage inadéquat des intrants chimiques dans la production et dans le stockage; (5) sur les Hautes Terres, une tendance des producteurs à focaliser leurs efforts sur les produits destinés au marché, au détriment de l'autoconsommation.

Les leviers proposés pour améliorer la durabilité du système alimentaire dans la zone 2 sont de: (1) structurer les filières partageant les mêmes intérêts et les échanges interprofessionnels tout au long de la chaîne pour limiter l'asymétrie de l'information et favoriser la qualité des produits, le contrôle de qualité, l'accès au marché et la traçabilité des produits et le stockage (en intégrant société civile, acteurs privés et acteurs publics pour la pérennisation du système);

(2) promouvoir la transformation et la conservation des produits, notamment par l'amélioration des infrastructures de stockage; (3) promouvoir l'éducation nutritionnelle et la sensibilisation à la consommation de produits de qualité, l'achat de proximité et la diversification des produits; (4) investir dans les infrastructures de commercialisation: marché de gros par district, centrales d'achat, remise en état des routes; (5) revaloriser le métier d'agriculteur et proposer des formations ciblées, accompagner les agroentrepreneurs émergents en proposant des études de marché, un meilleur accès au financement afin d'accroître leur intérêt vis-à-vis des produits de qualité; (6) préserver les ressources naturelles tout en améliorant la qualité des produits: élaboration de projets collectifs pour aborder la dégradation des ressources naturelles, promotion des "territoires à vocation bio" en rassemblant les acteurs concernés par les filières bio et la valorisation des terroirs, diffuser les Systèmes participatifs de garantie (SPG) pour engager les producteurs sur les bonnes pratiques et créer des liens entre consommateurs et producteurs au niveau des marchés de détail; (7) élaborer un code d'hygiène et des normes à respecter par les transporteurs et les restaurateurs afin de conserver l'hygiène et l'aspect des produits alimentaires; (8) mieux mettre en relation la production et la demande, favoriser des liens entre producteurs

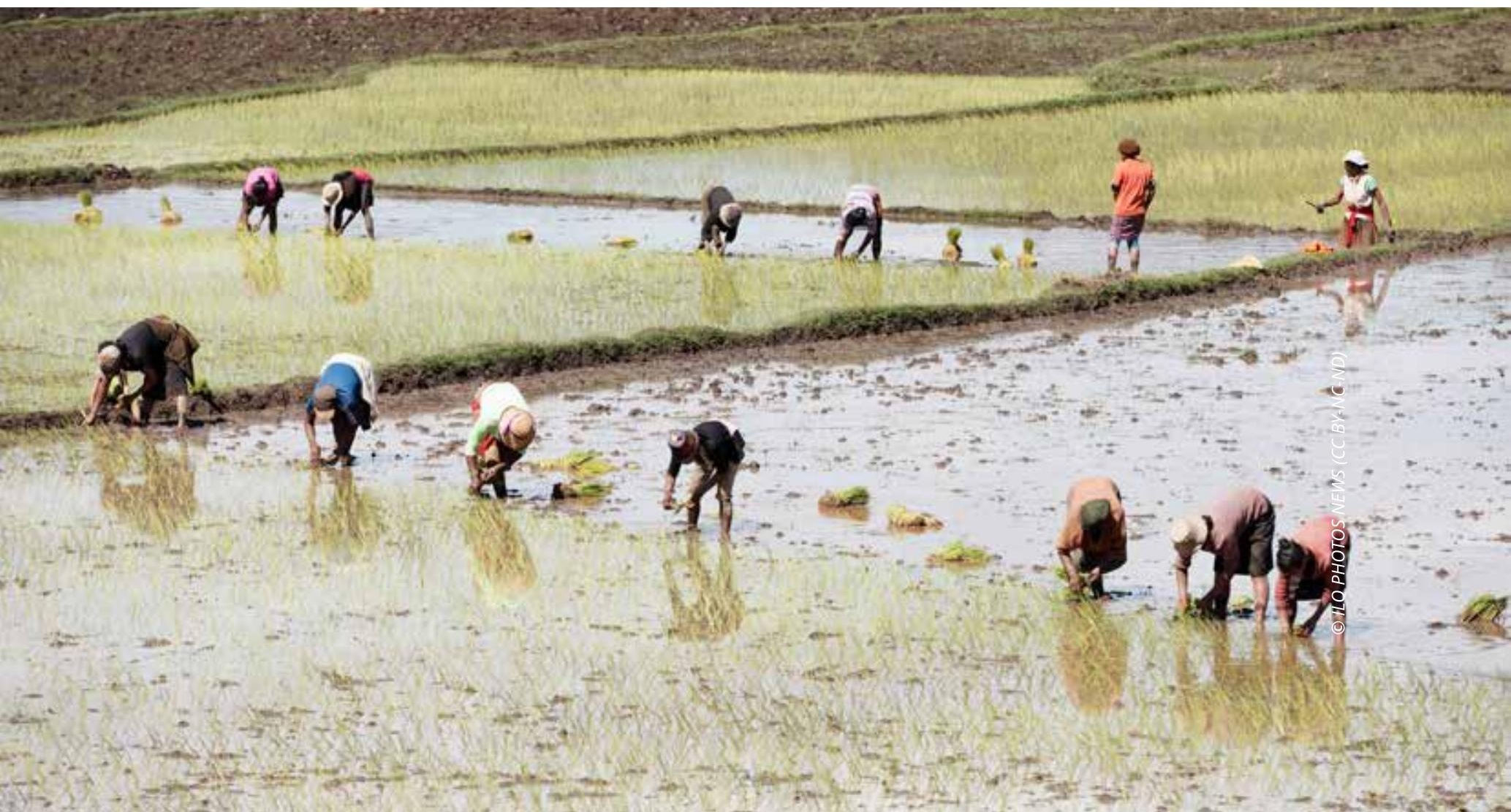


et consommateurs (fidélisation); (9) réfléchir à des politiques de régulation des prix pour limiter la volatilité des prix.

\section{Zone 3: le Grand Est}

Une zone de cultures industrielles et d'exportation

La zone 3 est située à l'est du pays et regroupe trois régions: Atsinanana, Analanjirofo et Vatovavy Fitovinany. 60 à 90 pour cent de la population est rurale et vit de l'agriculture. L'exploitation agricole est assumée par le propriétaire et les membres de sa famille ou parfois en location sous régime de fermage ou de métayage. Essentiellement de type familial, le mode de production est extensif, sans soins particuliers et à rendement faible. Les cultures de rente (Fénérive Est, Maroantsetra et Mananara Nord) occupent 63 pour cent des surfaces cultivées contre 34 pour cent pour les cultures vivrières (Maroantsetra et Fénérive est). Le girofle est la première culture de rente avec 68 pour cent des surfaces tandis que le café vient en seconde position avec 26 pour cent des surfaces cultivées. La vanille commence à être développée (5 pour cent environ). Dans le secteur fruitier, le litchi est en train de devenir une culture de rente avec des efforts conjugués pour le développement de la filière. Le vieillissement des plantations et le changement climatique menacent les cultures de rente. En outre, les producteurs côtiers sont des pêcheurs artisanaux généralement à destination locale.

Globalement, la production vivrière couvre cinq à six mois de l'année et les producteurs doivent s'approvisionner sur le marché pour leurs besoins restants. Cependant, les revenus issus des cultures de rente tendent à faire l'objet d'une dilapidation par surconsommation en période faste de récolte et poussent le producteur à l'endettement en période de soudure et bien souvent à hypothéquer la récolte de la saison suivante. Une partie des ménages s'adonnent à des activités non agricoles de services pour combler les lacunes de revenus. Les producteurs de cultures de rente sont souvent regroupés en coopératives pour faciliter l'écoulement des produits.

Cette zone constitue un important bassin de consommation et d'opportunités d'emplois non agricoles avec la présence du port de Toamasina.

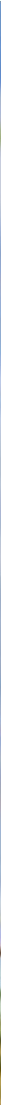


Les appuis autour des filières existent à travers les projets et programmes de développement qui y travaillent (PROSPERER, FRDA, etc.) même s'ils s'avèrent encore insuffisants. Les défis rencontrés pour cette zone sont: (1) l'instabilité financière des producteurs malgré les revenus significatifs issus des cultures de rente et de la pêche (le prix des produits de rente est très volatile et la répartition de la valeur ajoutée est plutôt en faveur des collecteurs, entreprises et usines de transformation; (2) la production alimentaire locale (maraîchère, etc.) ne permet pas d'assurer la demande car la population a tendance à la délaisser au profit des cultures de rente.

Les leviers identifiés pour équilibrer le système alimentaire de la zone sont: (1) la décentralisation: mieux définir les priorités régionales notamment pour ce qui est de l'équilibre entre les cultures vivrières et les cultures de rente et de la complémentarité avec les autres zones; (2) I'amélioration des infrastructures de communication (routes, voies ferrées, canal de Pangalanes); (3) la mise en place et l'entretien des infrastructures agricoles; (4) la mise en place des services agricoles de proximité (formations techniques et technologiques, gestion, accès financement, etc.); (5) l'appui aux organisations paysannes; (6) la promotion de la transformation agricole et l'amélioration des infrastructures de stockage; (7) la mise en relation entre producteurs et opérateurs pour une meilleure répartition de la valeur ajoutée (ex: agriculture contractuelle); (8) la promotion de l'éducation nutritionnelle en lien avec l'encadrement agricole (ex: jardin communautaire); (9) la promotion et l'initiation à l'hygiène alimentaire.

\section{Zone 4: le Grand Ouest}

Une zone à potentiel agricole élevé mais totalement enclavée, avec un taux de pauvreté élevé et avec une dégradation de l'environnement poussée

Géographiquement, cette zone rassemble trois régions: le Melaky, le quart occidental du
Bongolava et la frange Nord du Menabe. Espace agropastoral par excellence, cette zone sert également de plateforme de transit pour le bétail venant du sud et alimentant la zone 2 .

C'est une zone où les exploitations agricoles ont un revenu agricole moyen supérieur à la moyenne nationale. Mais il y a très peu de transformateurs agroalimentaires. La pêche industrielle d'export et la pêche artisanale à destination locale y sont présentes. L'aquaculture de crevette y est également pratiquée avec des investissements notables pour l'exportation de crevette de première qualité.

Cette zone abrite un site touristique et un paysage géologique unique de renommée mondiale qui est le Tsingy de Bemaraha (la forêt de pierre).

Les retombées du potentiel agricole sont globalement positives sur la sécurité alimentaire et la malnutrition des enfants mais les taux de malnutrition indiquent quand même des valeurs élevées révélant un problème de disponibilité et d'accès à la nourriture pour une partie de la population. Les autres défis sont: (1) une dégradation plus ou moins poussée des ressources naturelles à l'instar du sol, des ressources halieutiques et des forêts; (2) une répartition inéquitable de la valeur ajoutée agricole au détriment des producteurs et en faveur des collecteurs et commerçants; (3) un enclavement de la zone qui contribue fortement à la volatilité des prix; (4) une insécurité limitant le développement des activités relevant du système alimentaire.

Face à cela, les principaux leviers identifiés pour la zone 4 sont: (1) exploiter les potentialités actuelles pour la diversification agricole (légumes et fruits) et la promotion de la mécanisation agricole; (2) promouvoir la transformation ainsi que les infrastructures de stockage; (3) désenclaver la zone: remettre en état les routes (RN1 bis reliant Tsiroanomandidy et Maintirano) et exploiter le transport maritime entre Maintirano et Mahajanga pour améliorer ainsi l'accès au marché; (4) rétablir la sécurité publique; (5) 
promouvoir une éducation nutritionnelle basée sur l'utilisation des produits locaux; (6) mettre en place des actions de gestion durable des ressources halieutiques et forestières; (7) mettre en place les stratégies adéquates pour favoriser la répartition équitable de la valeur ajoutée tout au long de la chaîne; (8) favoriser des systèmes agrosylvopastoraux en organisant l'espace par rapport à la compétition agriculture-élevage créée par les transhumants.

\section{Zone 5: le Grand Sud}

Une zone d'élevage et de pêche caractérisée par l'avancée de la désertification accompagnée d'une insécurité alimentaire chronique et d'une migration marquée

La zone couvre cinq régions: Atsimo Andrefana, Ihorombe, Anosy, Androy et Atsimo Atsinanana. L'agriculture y est essentiellement pluviale et l'élevage de zébus et de petits ruminants prédomine. Sur le littoral, la pêche est à la fois artisanale pour le marché local mais également industrielle pour l'exportation (langouste dans la Région Anosy). La zone subit différents aléas climatiques: des épisodes récurrents de sécheresse frappent les régions arides et subarides tandis que l'Atsimo Atsinanana est régulièrement exposé aux cyclones et aux inondations. L'intensification du vent réduit les activités de pêche, accélère les phénomènes de formation de dunes et d'érosion éolienne. La présence de la chenille légionnaire et les problèmes phytosanitaires s'accentuent sous l'effet du changement climatique. En conséquence, la production vivrière locale est insuffisante aussi bien en quantité qu'en diversité (très peu de légumes et fruits) et les exploitations agricoles enregistrent de faibles revenus. Par ailleurs, la vétusté et le manque d'infrastructures agricoles, relatives à l'eau et aux télécommunications ainsi que l'insécurité publique limitent le développement des activités relevant du système alimentaire et l'enclavement contribue à la volatilité des prix des produits alimentaires. La transformation agroalimentaire demeure marginale réduisant ainsi la création de valeur ajoutée locale. Les activités minières industrielles et artisanales sont relativement importantes mais ces dernières sont faiblement rémunératrices pour les ménages concernés. En résultante, les taux de pauvreté et la vulnérabilité au changement climatique dans le Grand Sud sont parmi les plus élevés du pays, ne permettant pas à la population d'acheter de la nourriture en quantité et qualité requise une fois le stock épuisé, créant ainsi une situation d'insécurité alimentaire chronique accentué par les aléas climatiques. En effet, la zone compte plusieurs poches d'insécurité alimentaire modérée à sévère (affectant plus de 50 pour cent de la population), variant d'une année à l'autre mais qui concernent principalement les régions Atsimo Andrefana, Androy, Anosy et Atsimo Atsinanana. Face à l'insuffisance de la production aux conditions climatiques défavorables, et à l'insuffisance d'activités de subsistance durables, l'exploitation irrationnelle des ressources naturelles notamment forestières, halieutiques et minières constitue une activité génératrice de revenus complémentaires voire de survie et le phénomène de migration est particulièrement marqué.

Les leviers proposés pour la durabilité du système alimentaire du Grand Sud sont: (1) l'amélioration de l'accès à l'eau; (2) les actions visant à limiter l'avancée de la désertification et à assurer une gestion intégrée des ressources en eau, suivies d'aménagement et de mise en valeur durable des terrains agricoles potentiels; (3) le rétablissement de la sécurité publique; (4) la remise en état des routes afin de faciliter le flux des produits alimentaires et limiter la volatilité des prix; (5) l'éducation nutritionnelle des familles, à revisiter pour tenir compte des facteurs socio-anthropologiques; (6) l'amélioration de l'accès aux services de base (eau, électricité, santé et éducation); (7) l'amélioration des services agricoles; (8) le développement d'activités génératrices de revenus alternatives à l'agriculture et l'exploitation des ressources résilientes à la sécheresse; et (9) la coordination nationale et internationale des actions d'urgence et de la transition vers le développement. 


\section{Quelques leviers clés pour améliorer la durabilité du système alimentaire à Madagascar

\author{
Renforcer la décentralisation pour mieux définir les priorités régionales et \\ prendre en compte les complémentarités entre les zones
}

Les impacts attendus de sa mise en œuvre. Un renforcement adéquat de la décentralisation aurait des effets conséquents à plusieurs niveaux: (1) au niveau de l'environnement, une plus grande capacité locale à gérer les ressources naturelles dans le sens d'une optimisation durable du capital productif local, (2) au niveau politique, une accentuation et une priorisation des plans de développement établis au niveau local permettraient d'orienter les investissements publics vers la construction ou la réhabilitation des infrastructures infranationales pour désenclaver les territoires, (3) au niveau socioéconomique, la décentralisation effective facilite la mobilisation du secteur privé à s'implanter à l'échelle régionale, à canaliser positivement la migration démographique, à fiabiliser le système productif à travers des centres régionaux de développement, et enfin (4) au niveau de la consommation, une valorisation plus importante des acteurs et des produits locaux est rendue possible au niveau du maillon de consommation par le renforcement des techniques de transformation locale vers la promotion, par exemple, d'aliments «fortifiés».

Les conditions de réussite. Les effets positifs ci-dessus cités ne peuvent être concrétisés sans les conditions suivantes: (1) politique purement politicienne à proscrire dans les prises de décision: les mesures et programmes

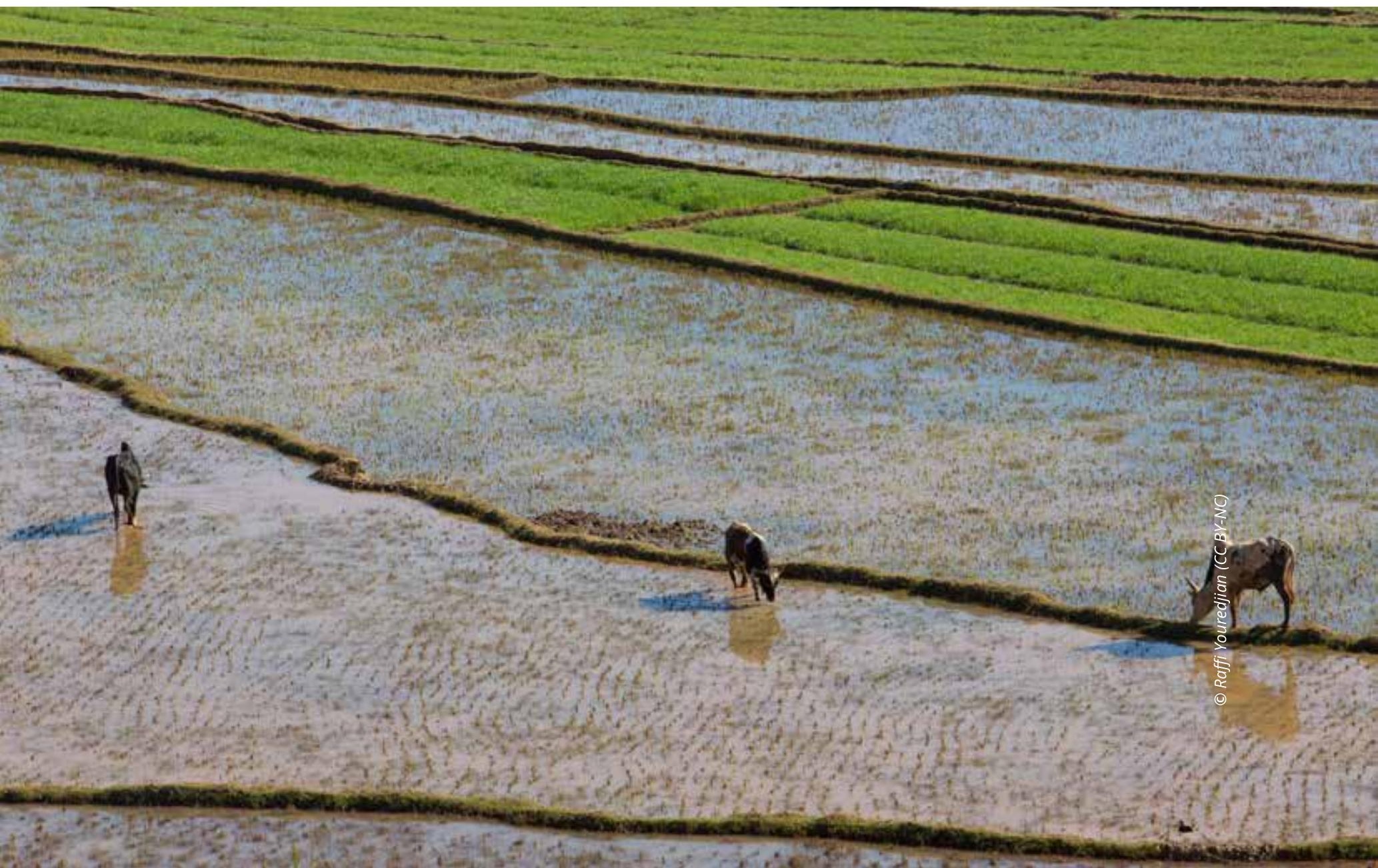


pour le développement local servent souvent de propagande et de clientélisme politiques, au détriment de la continuité et de la durabilité, (2) valorisation des capacités locales dans la gestion des ressources: le développement de compétence et d'expertise locales est nécessaire pour la capitalisation, l'appropriation et la continuité du développement de proximité,

(3) maîtrise par les élus/gouvernants locaux de la notion de sécurité alimentaire (IEC,...): le renforcement de capacité des collectivités territoriales décentralisées (CTD) est nécessaire pour maintenir les effets durables des décisions prises au niveau local ainsi que les interactions positives, (4) disponibilité à temps des intrants agricoles (par rapport au calendrier culturel): à défaut de flux tendus, l'approvisionnement des territoires doit se faire en temps réel et s'affranchir des lourdeurs liées à l'inertie administrative.
Les risques, les conflits d'intérêts et facteurs de blocage. (1) Une démotivation des paysans est un risque réel par rapport aux importations massives de denrées de base, soit à cause de la concurrence déloyale, soit que le manque de coordination entraîne une surproduction relative et une mévente, tandis que (2) l'absence ou l'insuffisance de transfert de pouvoir et de compétences au niveau régional et local exposent les territoires au manque de décision étatique sur les programmes/projets infranationaux d'envergure et à la pérennisation de monopoles particuliers d'intérêts.

\section{Les expériences fructueuses dans le}

\section{domaine... (1) La loi Gelose (gestion locale}

sécurisée) est un programme efficace de transfert et de gestion locale des ressources naturelles démarré dans les années 1990 entre l'Etat à travers le Ministère en charge des eaux

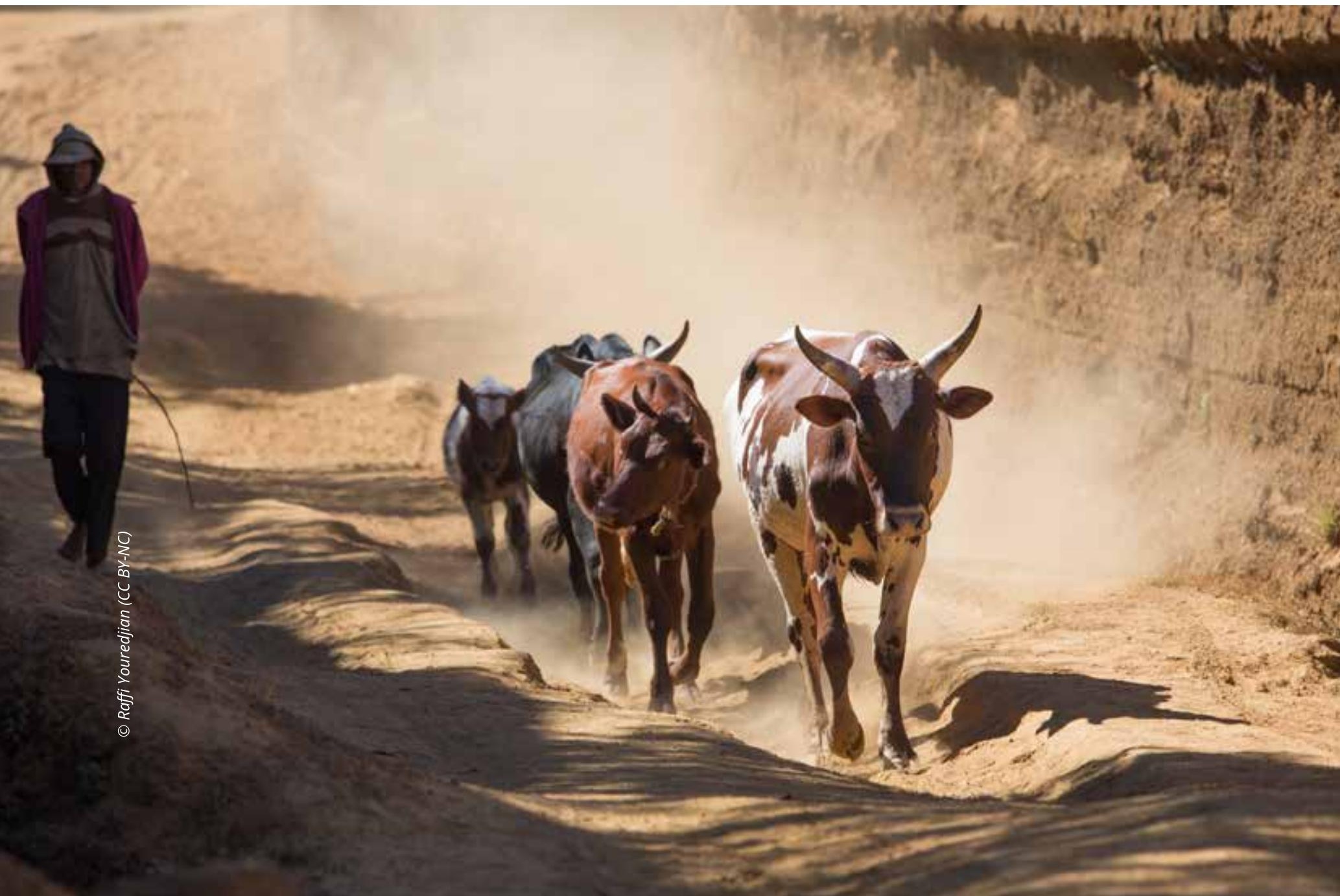


et forêts et trois catégories d'acteurs (1) locaux (communautés locales de base), (2) communaux (maires et conseils communaux) et (3) techniques (services déconcentrés chargés des forêts, des ressources pastorales ou halieutiques), tout en apportant une «Sécurité Foncière Relative» aux communautés de base. (2) L'agriculture contractuelle pour l'export ou l'approvisionnement de grandes entreprises apporte aux producteurs locaux la sécurité du marché et le transfert de savoir-faire à travers l'intérêt qu'offre leur terroir aux investisseurs du secteur privé. (3) La coopération décentralisée génère des partenariats entre les régions de Madagascar et les collectivités décentralisées de l'extérieur sous forme d'assistance technique pour l'amélioration de la productivité agricole.

\section{Mieux gérer la fertilité des sols pour assurer la qualité et la quantité des produits agricoles}

Les impacts attendus de sa mise en œuvre... Améliorer la gestion de la fertilité des sols (par l'utilisation des techniques biologiques et mécaniques de défense et de restauration des sols: [DRS]), agit sur plusieurs dimensions: (1) au niveau de l'environnement, elle permet de diminuer les ensablements dans les rizières notamment dans les bassins de production. Il est évident que la gestion des sols a des liens étroits avec la biodiversité (micro/macrobiodiversité du sol et autres), l'adoption des pratiques agroécologiques permet de restaurer les matières organiques et l'écologie du sol pour pouvoir augmenter la fertilité et la stabilité des sols, respectivement afin d'augmenter la productivité (moins de recours aux tavy) et afin d'éviter les érosions;

(2) au niveau socioéconomique, la gestion de la fertilité des sols permet de mieux valoriser les terrains de culture (extension et intensification) afin de mieux produire et de mieux gagner par rapport aux revenus, toutefois la cherté des fertilisants et la faible présence des services agricoles rendent l'accès à la gestion de la fertilité des sols difficile; (3) au niveau de la production, la relation est évidente car une meilleure gestion de la fertilité des sols ne peut qu'accroître la productivité et la production en conséquence; (4) au niveau de la consommation et la nutrition, la qualité nutritionnelle des produits pour la consommation dépend significativement de la qualité chimique des sols.

\section{Les conditions de réussite... Quelques} conditions sine qua non pour que la mise en œuvre soit effective: (1) une intervention par zone: il s'agit de faire des recherches pour connaître les sols de chaque terroir (carte de fertilité) pour pouvoir agir (méthodologie de fertilisation); (2) des politiques (sous)sectorielles claires et cohérentes entre elles: il s'agit d'avoir un cadre clair d'intervention précisant le rôle de chaque acteur, ses missions et les complémentarités avec les autres secteurs; (3) un accès aux services agricoles de proximité: nécessaire pour accéder aux fertilisants et pour bien les utiliser; (4) une association des éleveurs dans le processus: la gestion des feux et l'intégration agriculture élevage permet de mieux appréhender les problèmes de matières organiques. (5) un positionnement politique vis-à-vis de la fertilisation (organique, chimique ou mixte) et la considération des effets à long terme conditionnant la durabilité et les trajectoires des systèmes de production et donc des futurs systèmes agroalimentaires. Ainsi il faudrait accompagner la mise en œuvre effective des outils législatifs comme celui sur «l'agriculture biologique»

\section{Les risques, les conflits d'intérêts et}

\section{facteurs de blocage... (1) Les intérêts} divergents et conflits d'intérêts entre agriculteurs et éleveurs surtout dans les zones étendues constituent un risque majeur qui pourrait bloquer la mise en œuvre de ce levier; (2) suivant les objectifs de chaque producteur, il existe deux voies possibles sur lesquelles des débats ont encore lieu à ce jour: la voie agroécologique à promouvoir 
pour assurer la durabilité et la voie de l'intensification basée sur les intrants chimiques.

Les expériences fructueuses dans le domaine... Les expériences réussies dans le domaine sont surtout celles du GSDM (Groupement Semis direct de Madagascar) dans l'intensification agroécologique et dans l'utilisation des techniques agroforestières, en particulier dans l'utilisation des plantes de services associées notamment le Mucuna et d'autres légumineuses. Les initiatives des entités qui promeuvent la valorisation des déchets organiques sont à développer.

\section{Intensifier le renforcement des services de proximité pour tous les acteurs}

Les impacts attendus de sa mise en œuvre... (1) Renforcement des services de proximité pour les producteurs et les acteurs en aval, ce qui permet d'agir à plusieurs niveaux. (2) Limitation de la dégradation des sols et de la déforestation en accompagnant les producteurs vers la maîtrise de pratiques d'intensification durable, comme les techniques agroécologiques. (3) Amélioration, par ces mêmes pratiques et par la réduction de l'utilisation d'intrants chimiques, de la qualité sanitaire des produits (en particulier pour le maraîchage). L'action conjointe du conseil agricole, de l'accès au crédit, de la disponibilité d'intrants et des semences adaptés, permet d'augmenter la qualité et la diversité des produits, ce qui joue en faveur des revenus des producteurs et de l'offre alimentaire pour les consommateurs. (4) Un accès facilité au crédit stimule également le stockage et la transformation, avec une incidence sur les revenus des acteurs, comme sur la régularité et la diversité de l'offre.

\section{Plusieurs conditions apparaissent} fondamentales pour que ce levier soit efficace: (1) renforcer la place de la recherche et son adaptation au savoir traditionnel dans le conseil/formation à travers une meilleure articulation entre recherche et services de proximité, (2) promouvoir une position d'accompagnement-conseil et non de prescription d'imposition, (3) associer conseil et financement au niveau communautaire, (4) définir les attributions des différents acteurs (État, organisations de producteurs, privés), (5) développer une approche intégrée entre l'agriculture, l'élevage et la santé, (6) capitaliser les expériences réussies en développement de services de proximité en tirer les enseignements, (7) formaliser les secteurs informels et améliorer la sécurité d'investissement tout en valorisant les investisseurs locaux.

Les risques, conflits d'intérêts et facteurs de blocage... La discontinuité des programmes constitue un risque important pour le renforcement des services de proximité alors que les changements se font dans la durée. Les dispositifs portés par les paysans eux-mêmes sont parmi les plus efficaces mais la faible reconnaissance par les producteurs eux-mêmes de ces dispositifs (difficultés à accepter de compenser les services fournis) ainsi que la perception selon laquelle les services doivent être entièrement pris en charge par l'Etat ou les projets s'érigent en facteurs de blocage.

Expériences fructueuses. Le conseil agricole porté par des conseillers agricoles de proximité en collaboration avec des paysans relais, la multiplication des semences, les pépinières villageoises peuvent être cités parmi les services de proximité intéressants à développer.

\section{Favoriser la bonne organisation des chaînes de valeur agricoles et/ou alimentaires}

Les actions possibles et les impacts attendus. La coordination des actions entre les producteurs, les commerçants et les acheteurs pourrait se faire de différentes manières et serait bénéfique à plusieurs égards: (1) la structuration des 
producteurs, leur connexion avec le secteur privé constituent également une porte d'entrée pour améliorer la valeur ajoutée au niveau des producteurs; (2) la diversification de la production réduirait la compétition entre les producteurs; (3) la mise en marché de produits biologiques et agroécologiques permettrait de réduire la pollution de l'environnement (eau, sol), d'assurer la fourniture d'aliments plus sains pour les consommateurs à travers des mécanismes de garantie et de mettre en valeur certains territoires; (4) la conservation et la transformation des aliments diminueraient les pertes et stimuleraient l'économie locale; (5) la valorisation des aliments endogènes.

\section{Les conditions de la réussite. Cette}

organisation des chaînes de valeur requiert cependant des préalables: (1) une meilleure gouvernance locale; (2) le pilotage de l'État et un partage des responsabilités; (3) un dialogue multi-acteurs et multi-niveaux des parties prenantes, y compris les consommateurs et communautés, ainsi qu'une gestion foncière plus appropriée; (4) la création d'une dynamique d'appropriation de la communauté.

\section{Les risques, conflits d'intérêts et facteurs} de blocage. Les risques sont nombreux dont:

(1) l'absence d'une vision cohérente au sein de l'Etat (par exemple pour la production biologique, existence d'un noyau dur prônant une agriculture intensive en intrants chimiques);

(2) la diversité des intérêts y compris au niveau des institutions; (3) le cloisonnement entre les acteurs; (4) la gestion de la politique de commercialisation du riz, dont la priorité est le prix aux consommateurs.

Parmi les expériences fructueuses figure le Système participatif de garantie pour les fruits et légumes agroécologiques développé en périphérie d'Antananarivo avec l'appui du programme ASA (UE).

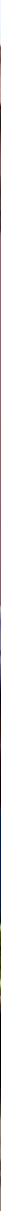




\section{Améliorer durablement les infrastructures de transport dans les zones agricoles potentielles}

Les impacts de sa mise en œuvre... Ce levier permet d'accroître l'accès aux différents services de base (santé, services agricoles...) et de faciliter l'acheminement des produits. II facilite l'instauration de la sécurité, encourage les investissements extérieurs à l'implantation, favorise le marketing territorial et permet un accès à des produits de plus en plus diversifiés. Pour ce qui est de l'environnement, l'amélioration des voies de communication est à double tranchant car elle permet à la fois de favoriser la gestion durable des ressources naturelles et d'entrainer la déforestation.

Les conditions de la réussite... Les questions relatives au financement sont essentielles pour le désenclavement. La planification en termes d'investissements prioritaires est un rôle régalien de l'Etat. Mais la planification aux échelles régionales et locales, les questions foncières, la gestion durable des ressources naturelles, la coordination des actions, la participation locale sont autant nécessaires pour que les actions puissent être efficaces. La considération des liaisons entre zones de production et avec les centres de consommation est prioritaire.
Les risques et conflits d'intérêt... Le retour sur investissement est lent, c'est une activité à long terme. Le désenclavement ne profite pas à tous les acteurs, certains acteurs ont un intérêt dans le maintien de l'enclavement pour pouvoir pérenniser leurs investissements. Certains bassins de production majeurs restent ainsi enclavés du fait de différents lobbies économiques et politiques. En effet, le désenclavement a, de tout temps, été une question politique surtout en période électorale.

Expériences fructueuses... Les péages (ONG Lalana), ont permis de financer les travaux d'entretien des routes par les collectifs d'usagers. L'utilisation des matériels biologiques à l'exemple des talus biologiques pour prévenir les risques de dégradation des infrastructures. Les actions des unions de paysans ont permis la gestion des équipements de transport à petite échelle et de réhabiliter les pistes: les kubota ont été utilisés pour transporter les produits.

Ces leviers constituent des pistes d'interventions qui nécessitent d'être précisés, par un dialogue plus approfondi entre les différents secteurs concernés et les acteurs du système, afin d'aller vers un système alimentaire plus durable. 


\section{Méthodologie au niveau national et processus}

Cette note est issue d'une collaboration entre le Gouvernement de Madagascar représenté par le Ministère de l'agriculture, de l'élevage et de la pêche (MAEP), le Ministère de l'environnement et du développement durable (MEDD) et le Ministère de l'industrie, du commerce et de l'artisanat (MICA), coordonnés par le MAEP, I'Union européenne, la FAO et le Cirad. Elle s'inscrit dans l'initiative Activer la transformation durable et inclusive des systèmes alimentaires.

La méthode d'analyse combine des données quantitatives et qualitatives et une approche participative impliquant les acteurs publics et privés liés aux systèmes alimentaires. La littérature et les données disponibles, ainsi que des entretiens auprès de personnes ressources, ont permis d'approfondir les processus à l'œuvre et d'affiner la compréhension systémique.

Des réunions hebdomadaires avec les parties prenantes ont permis de partager et d'affiner les résultats et d'identifier les principaux leviers à actionner pour améliorer la durabilité du système.
L'approche systèmes alimentaires (SA) prend en compte les différents objectifs des SA, le large éventail d'acteurs impliqués, les moteurs et les mécanismes de gouvernance qui façonnent leurs activités. En cela, elle est holistique et multidimensionnelle. Enfin, elle est systémique car elle met l'accent sur les interactions, rétroactions, synergies et compromis entre les différentes composantes des SA. Elle se différencie des approches traditionnelles qui ont tendance soit à être sectorielles et à ne considérer que certaines dimensions des SA, soit à se limiter à l'analyse systémique de certains segments de la chaîne d'approvisionnement (par exemple, les systèmes de production).

La performance du système alimentaire est évaluée au regard des quatre objectifs de durabilité des systèmes alimentaires: i) sécurité alimentaire, nutrition et santé, ii) durabilité socioéconomique, iii) durabilité environnementale et iv) équilibre territorial et équité. Les systèmes alimentaires durables sont un point d'entrée pour 12 des 17 ODD.

Figure 2. Cadre analytique

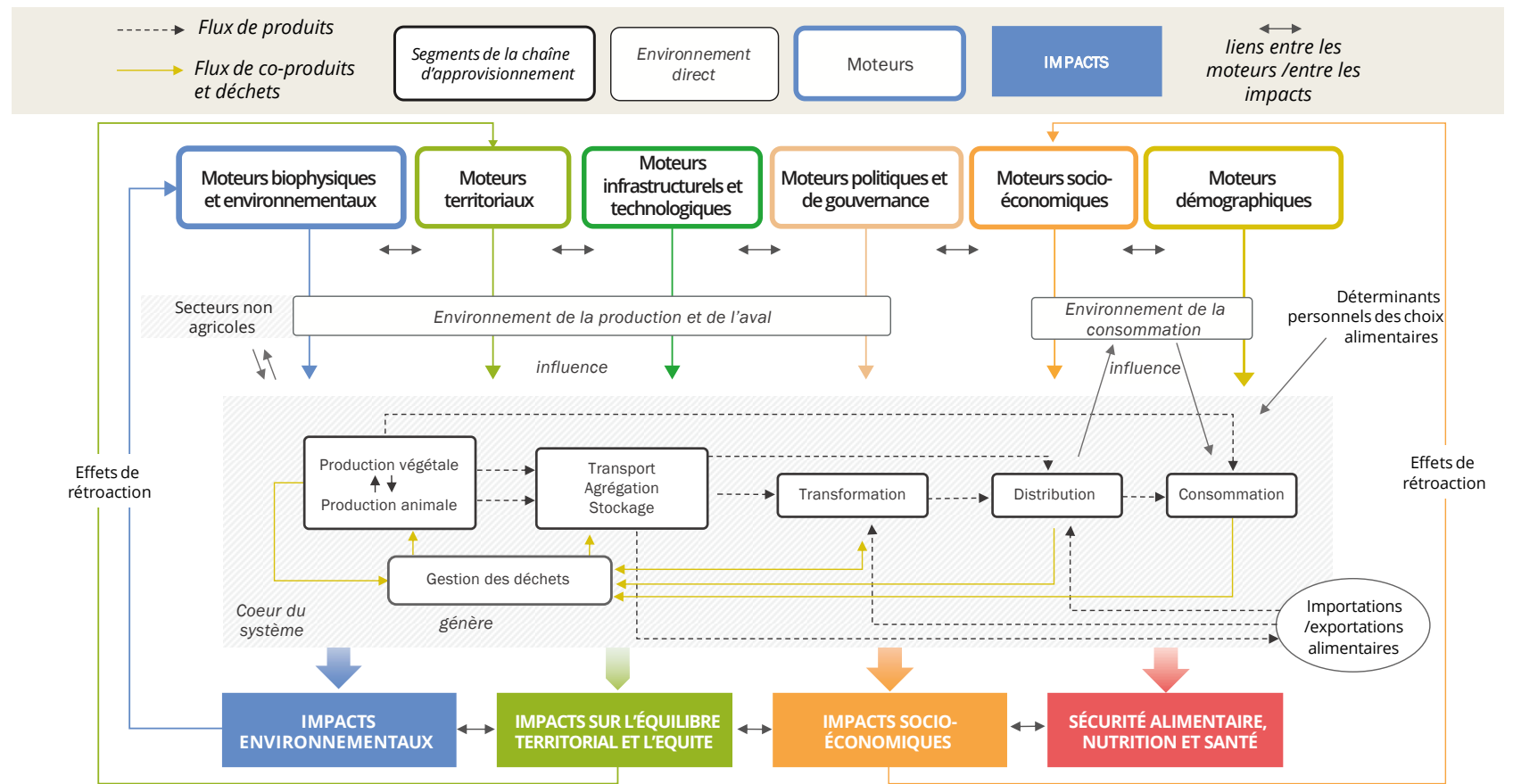

Source: les auteurs. 


\section{Références}

Banque mondiale. 2016. Agriculture et développement rural à Madagascar - Background Papers. Washington.

Banque mondiale. 2019. Madagascar. Note de conjoncture économique. Un nouveau départ? Washington.

FEWS NET. 2020. Madagascar. Bulletin des prix janvier 2020.

INSTAT. 2010. Enquêtes périodique auprès des ménages 2010. Antananarivo. www.instat.mg/p/epm-enquete-periodique-aupres-des-menages-2010

MAEP, FAO, PAM. 2019. Évaluation de la production agricole et de la sécurité alimentaire à Madagascar.

Rabefarihy A.T. 2013. Identification et modélisation des facteurs socioéconomiques de déforestation et de dégradation forestière à Madagascar: application à la recherche de mesures incitatives adéquates dans le cadre de la mise en place de REDD+ - Thèse de Doctorat en sciences agronomiques. Option Eaux et Forêts. Antananarivo.

Ont contribué à ce processus et à l'élaboration de cette note:

A.T. Rabefarihy, H. Raharinjanahary, H.M. Tsimisanda (consultants); T.Randriarilala (FAO/Madagascar); T. Rakotomavo, F. Urena-Lara (UE/Madagascar); H.David-Benz, C.Orbell, N.Sirdey (Cirad); P.Herlant, J.Tefft (FAO/CFI).

Mise en page et édition: Chiara Virdis, Dominique Magada et Bruno Batreau. 


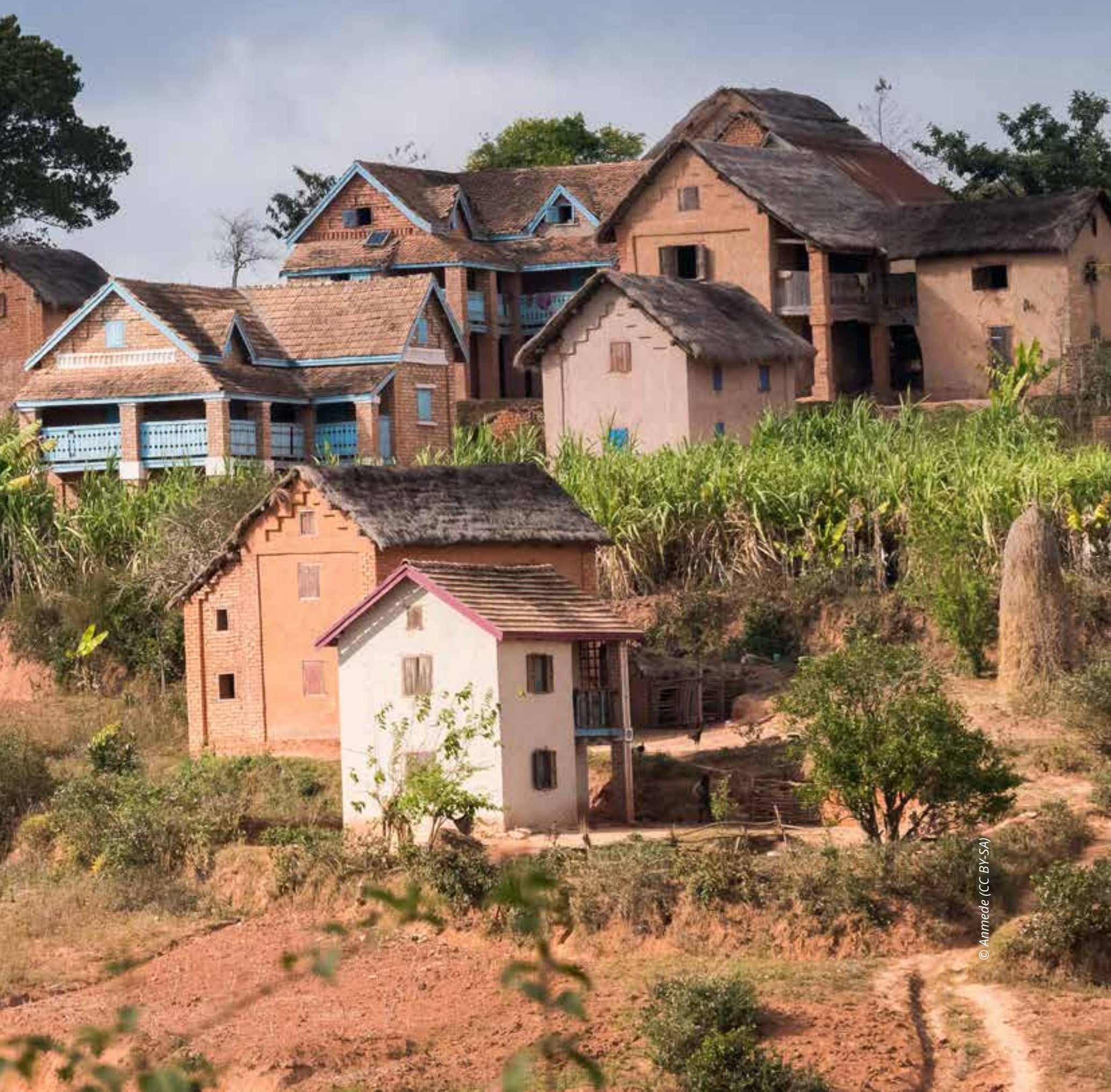




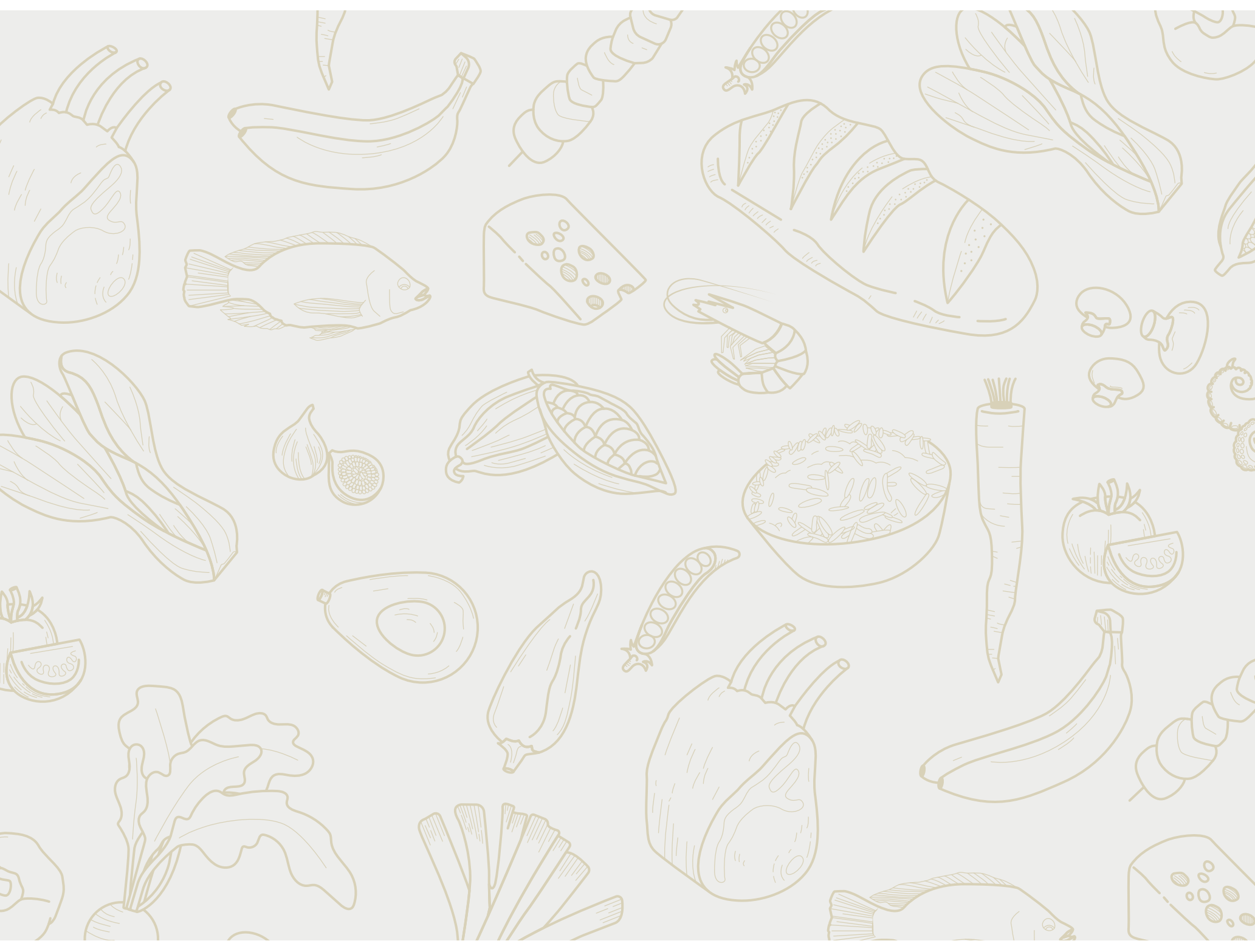

